Effects of Ion-Ion Collisions and

Inhomogeneity in Two-Dimensional Kinetic Ion Simulations of Stimulated Brillouin Backscattering

B. I. Cohen, L. Divol, A. B. Langdon, E. A. Williams

October 18, 2005

Physics of Plasmas 
This document was prepared as an account of work sponsored by an agency of the United States Government. Neither the United States Government nor the University of California nor any of their employees, makes any warranty, express or implied, or assumes any legal liability or responsibility for the accuracy, completeness, or usefulness of any information, apparatus, product, or process disclosed, or represents that its use would not infringe privately owned rights. Reference herein to any specific commercial product, process, or service by trade name, trademark, manufacturer, or otherwise, does not necessarily constitute or imply its endorsement, recommendation, or favoring by the United States Government or the University of California. The views and opinions of authors expressed herein do not necessarily state or reflect those of the United States Government or the University of California, and shall not be used for advertising or product endorsement purposes. 


\title{
Effects of Ion-Ion Collisions and Inhomogeneity in Two-dimensional Kinetic Ion Simulations of Stimulated Brillouin Backscattering
}

\author{
B.I. Cohen, L. Divol, A.B. Langdon, and E.A. Williams \\ University of California Lawrence Livermore National Laboratory \\ P.O. Box 808, Livermore, CA 94551
}

Two-dimensional simulations with the BZOHAR [B.I. Cohen, B.F. Lasinski, A.B. Langdon, and E.A. Williams, Phys. Plasmas 4, 956 (1997)] hybrid code (kinetic particle ions and Boltzmann fluid electrons) have been used to investigate the saturation of stimulated Brillouin backscatter (SBBS) instability including the effects of ion-ion collisions and inhomogeneity. Ion-ion collisions tend to increase ion-wave dissipation, which decreases the gain exponent for stimulated Brillouin backscattering; and the peak Brillouin backscatter reflectivities tend to decrease with increasing collisionality in the simulations. Two types of Langevin-operator, ion-ion collision models were implemented in the simulations. In both models used the collisions are functions of the local ion temperature and density, but the collisions have no velocity dependence in the first model. In the second model, the collisions are also functions of the energy of the ion that is being scattered so as to represent a Fokker-Planck collision operator. Collisions decorrelate the ions from the acoustic waves in SBS, which disrupts ion trapping in the acoustic wave. Nevertheless, ion trapping leading to a hot ion tail and two-dimensional physics that allows the SBS ion waves to nonlinearly scatter remain robust saturation mechanisms for SBBS in a high-gain limit over a range of ion collisionality. SBS 
backscatter in the presence of a spatially nonuniform plasma flow is also investigated.

Simulations show that depending on the sign of the spatial gradient of the flow relative to the backscatter, ion trapping effects that produce a nonlinear frequency shift can enhance (auto-resonance) or decrease (anti-auto-resonance) reflectivities in agreement with theoretical arguments.

PACS: 52.38.-r 52.65.Rr 


\section{INTRODUCTION AND MOTIVATION}

There is continuing interest in the nonlinear interaction of intense, coherent electromagnetic waves with high-temperature fusion plasmas. Nonlinear laser-plasma interactions are a significant concern for the use of intense lasers in inertial confinement fusion (ICF). This study addresses physics affecting the nonlinear saturation of stimulated Brillouin scattering (SBS), a process in which laser light is scattered by an ion acoustic wave resonantly excited by the ponderomotive force of the incident and scattered electromagnetic waves.

This work extends work published in Refs. 1 and 2 by including the separate influences of ion-ion collisions and a spatially inhomogeneous plasma flow in twodimensional simulations of the nonlinear saturation of stimulated Brillouin backscatter (SBBS). One-dimensional (1D) and two-dimensional (2D) hybrid (particle ions and Boltzmann fluid electrons) simulations have been performed previously with the BZOHAR simulation code investigating the saturation of SBBS in a homogeneous plasma slab as described in Refs. 1 and 2. It was observed that ion trapping, wave breaking, pump depletion, two-ion-wave decay instability and nonlinear ion Landau damping, harmonic generation, and driven ion-acoustic turbulence contribute to the saturation of SBBS. The principal result in Ref. 2 was that SBBS reflectivities saturate at significantly lower amplitudes in the 2D simulations than do SBBS reflectivities in 1D simulations. Ref. 2 showed that the decay of the SBBS ion wave (IAW) into longer wavelength ion waves and quasi-modes (induced scattering by ions) in 2D is active during the crash of the SBBS. ${ }^{1-5}$ After the SBBS crash in 2D the ion velocity distribution supports substantial ion wave dissipation: it has a significant population of ions in the tail 
and is less flattened and not as inverted as in 1D. Ion mode coupling in 2D can provide additional dissipation when the ion waves have finite amplitude. There is evidence of self-focusing or filamentation and forward SBS for $Z T_{\mathrm{e}} / T_{\mathrm{i}} \gg 1$ after SBS backscatter saturates in the 2D simulations reported in Ref. 2. Because the SBS backscatter occurs early in the simulation, saturates, and crashes, the backscatter is largely independent of the subsequent forward scattering and self-focusing or filamentation.

Earlier work on the theory and simulation of SBS has shown the importance of wave breaking and ion trapping, ${ }^{1-4,6-12}$ harmonic generation, ${ }^{13-15}$ and secondary instability of the ion wave. ${ }^{1-5}$ In $1 \mathrm{D}$ simulations of SBBS, the inclusion of kinetic electrons has made a difference in the saturation of SBBS. ${ }^{16,17}$ Rambo, Wilks, and Kruer undertook hybrid simulations (fluid electrons and particle-in-cell ions) of SBBS including ion-ion collisions in one spatial dimension. ${ }^{18}$ They observed that collisions affect the ions resonant with the SBBS ion waves so as to inhibit the flattening of the ion velocity distribution function due to trapping and thermalize the ion wave energy and the hot ion tail into the bulk ion velocity distribution. By maintaining slope in the ion velocity distribution, the collisions serve to maintain the ion wave damping closer to its linear value. Moreover, heating of the bulk ions by collisions reduces the value of $Z T_{\mathrm{e}} / T_{\mathrm{i}}$, which increases ion wave damping.

Here we study the effects of ion-ion collisions on SBBS in simulations undertaken in two spatial dimensions. For a fixed value of $Z T_{\mathrm{e}} / T_{\mathrm{i}}$ we performed a series of simulations varying the relative ion collisionality. We observe that peak SBBS reflectivities are reduced with increasing ion-ion collisionality and that the saturation is qualitatively similar to the collisionless results in Ref. 2 in most respects. The saturation 
of high-gain SBBS in 2D is dominated by ion trapping that produces hot ion tails, transient harmonic generation, and a temporal crash of the backscatter that is marked by nonlinear scattering of the SBS ion waves into longer-wavelength, obliquely propagating ion waves and ion quasi-modes (heavily damped by ions) in most cases. These simulations results and those reported in Refs. 1 and 2 support recent experimental observations of the importance of ion trapping effects ${ }^{19,20}$ and the two-ion-wave decay ${ }^{21,22}$ associated with SBS.

In addition, we study the nonlinear saturation of SBBS in two dimensions including a spatially inhomogeneous plasma flow. In qualitative agreement with Ref. 11, our simulations indicate that if ion trapping nonlinearities are sufficiently strong, i.e., if the ion-wave nonlinear frequency shift due to ion trapping is comparable to or larger than the ion-wave dissipation rate, then SBBS reflectivities are enhanced by auto-resonant effects if the spatial gradient of the flow is parallel to the backscatter direction; and the reflectivities are decreased by anti-auto-resonant effects if the spatial gradient of the flow is anti-parallel to the backscatter direction. As in Ref. 2, we focus on SBS ion-wave nonlinearities associated with kinetic ions in a two-dimensional model in which electron kinetic effects are deliberately omitted, which reduces the complexity of the physics and facilitates performing relatively large 2D simulations and undertaking systematic parameter scans.

The rest of the paper is organized as follows. Section II briefly reviews the BZOHAR simulation model and the two distinct collision algorithms that have been implemented along with a few test cases. In Section III we present simulations of stimulated Brillouin scattering and nonlinear forward scattering, and accompanying 
analysis and interpretation. SBBS simulation results including a spatial gradient in the plasma flow are reported in Section IV. Conclusions are given in Sec. V.

\section{SIMULATION MODEL WITH ION-ION COLLISIONS}

\section{A. BZOHAR Hybrid Simulation Model}

BZOHAR uses an efficient 2D hybrid simulation model with particle ions and Boltzmann fluid electrons. ${ }^{1,2}$ A temporal-enveloped wave equation with fluid electron current determines the electromagnetic vector potential which is linearly polarized perpendicular to the simulation plane. Poisson's equation with Boltzmann electrons to remove fast electron time scales yields the electric potential $\square$ where the total potential seen by the electrons is $\square(\vec{x}, t)=\square(\vec{x}, t)+\frac{m_{e}}{2}\left|e \tilde{A}_{z} / m_{e} c\right|^{2}$ and the electron density is given by $n_{e}=n_{0} e^{e \square / T_{e}}$ Multiple species ions are advanced as superparticles with nonrelativistic Newtonian equations of motion, and the ions only respond to the longitudinal electric field $\operatorname{LI} \square(x, y)$ appropriate to the limit $m_{\mathrm{e}} / m_{\mathrm{i}}<<1$. The incident laser propagates in $x$ from left to right and enters the plasma at $x=0$. The boundary conditions are periodic in $y$ and open/bounded in $x$ (outgoing electromagnetic waves, $f_{\mathrm{s}}=\square=0$ at $x=0$ and $x=L_{x}$, and the ions are confined by an imposed potential barrier). No backscatter electromagnetic seed is used here; the scattered electromagnetic waves grow from noise deriving from ion particle discreteness that serves as a source for charge density fluctuations and hence electron current fluctuations in the wave equation.

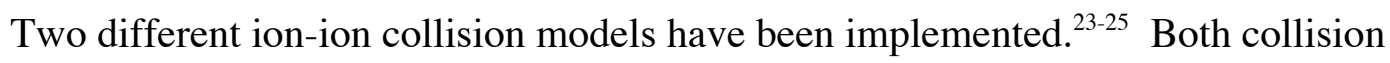
models are based on Langevin models wherein drag and diffusion increments to the ion velocities are computed in Monte Carlo fashion using moments of the local ion velocity distribution functions accumulated on the grid (spatially local density, mean drift 
velocity, and temperature). Drag-diffusion coefficients are calculated in the local drift frame using a characteristic collision frequency that depends on the local density and temperature. ${ }^{23,25}$ The collision operators approximately conserve energy and momentum in the limit of many particles per cell and many small-angle collisions per characteristic collision time. Particles are conserved trivially.

\section{B. Velocity-independent Collision Operator}

The first collision scheme employed uses the algorithm introduced by Jones, et $a l .^{23}$ Jones, et al. devised a grid-based, Langevin collision operator for interspecies and intraspecies Coulomb collisions. Their like-particle Langevin collision operator has drag and diffusion components:

$$
\frac{d}{d t}(\mathbf{v})_{c o l l}=\square(\mathbf{v} \square<\mathbf{v}>)+\mathbf{A}
$$

where

$$
\square=\square_{0}=\frac{4 \square^{1 / 2} n Z^{4} e^{4} \ln \square}{3 m^{2}(T / m)^{3 / 2}}
$$

and $\mathbf{A}$ is a random vector function distributed isotropically with probability

$$
P(A \square t)=\left(\frac{m}{4 \square \square \square T}\right) \exp \frac{m \mathbf{A} \cdot \mathbf{A} \square t^{2}}{4 \square \square t T}=
$$

defined for an implementation in two velocity dimensions. Thus,

$$
\mathbf{A} \square t=2<\mathbf{v} \cdot \mathbf{v}>^{1 / 2}(\square t)^{1 / 2}[\square \ln (1 \square \operatorname{ranf}(1))]^{1 / 2}[\cos \square, \sin \square], \square=2 \square \square \operatorname{ranf}(2),
$$

where $<\mathbf{v}>$ and the density $n$ and temperature $T$ in $\square$ are calculated locally by interpolating to the grid and back to the particles using linear area weighting (cloud-incell, CIC). The algorithm statistically conserves momentum and energy (through first order in $\square t$ ). To stabilize a cooling instability in the algorithm due to finite sampling statistics, ${ }^{23,26}$ we replaced the local $\mathrm{v}_{\mathrm{th}}=(T / m)^{1 / 2}$ in the denominator of $\square$ with $\mathrm{v}_{\mathrm{th}}{ }^{*}=\nabla_{\mathrm{th}}+(1-$ 
$\square \mathrm{v}_{\mathrm{th}}(\mathrm{t}=0)$. We find that with $[-0.9-0.97$ there is no cooling instability, and the local collision frequency can evolve in space and time in 2D BZOHAR simulations.

To test the implementation of the Jones, et al. collision algorithm we undertook three test simulations. The first test was whether the collision algorithm preserved a Maxwellian ("first do no harm") shown in Fig. 1. A 2D Maxwellian was initialized, and the plasma was allowed to evolve collisionally over $2000 \square t$ with $N_{\text {cell }}=100, \square \square \square t=0.001$ and no laser field present. The collision operator was applied every $10 \square t$. Kinetic energy and momentum conservation errors associated with the collision algorithm were $<1 \%$, and the ion velocity distribution function remained Maxwellian. With nearest-grid-point (NGP) interpolation used in the collision algorithm kinetic energy and momentum conservation errors were worse $(<3 \%)$. There was $\sim 6 \%$ self-
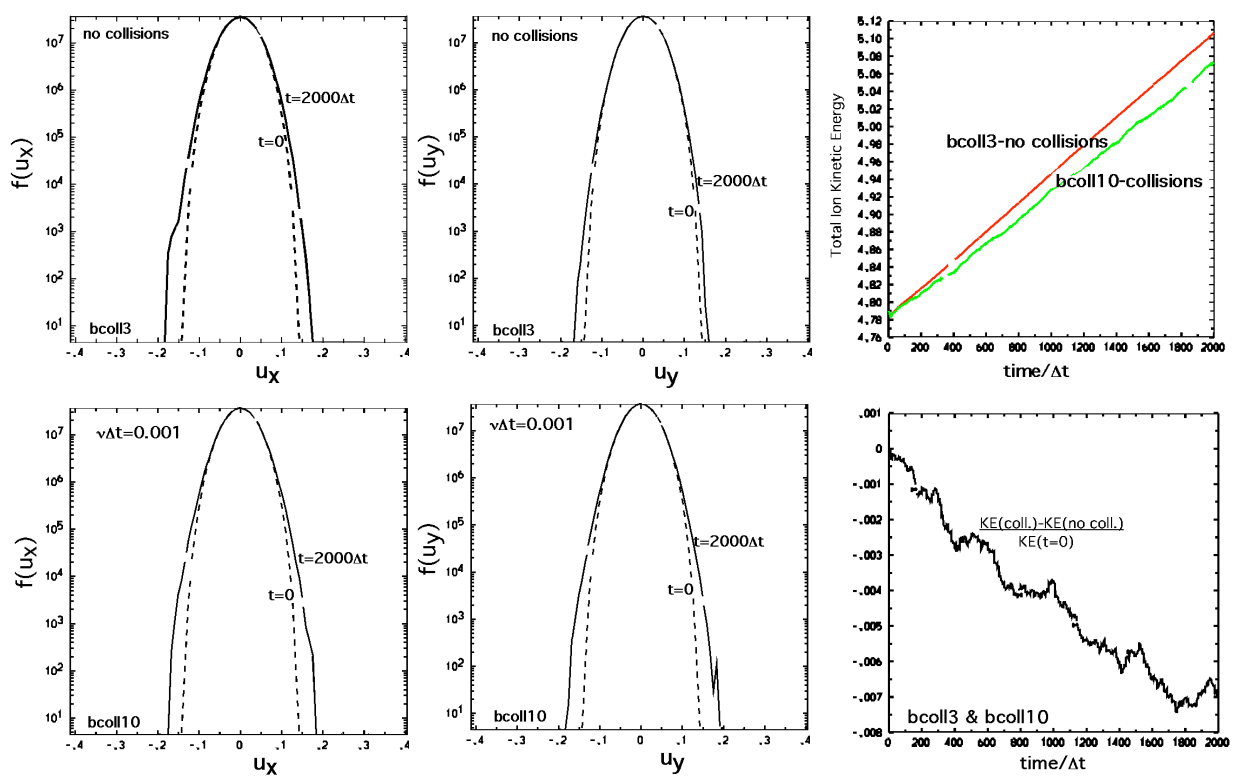

Figure 1. Preservation of a Maxwellian: comparison of evolved ion velocity distribution functions with no collisions (bcoll3) and with CIC collisions $\square_{b} \square t=0.001$ (bcoll10) showing that the Maxwellian is preserved and there is similar self-heating. 
heating with or without collisions. With 3.2M ions and $N_{\text {cell }}=100,2000$ time steps corresponded to $6100 \mathrm{CPU}$ sec with CIC collisions and 1700 secs with no collisions.

We next undertook simulations testing whether a Maxwellian was preserved with $N_{\text {cell }}=16$, the test-particle number density employed in our SBS simulations. Again a 2D Maxwellian was initialized, and the plasma was allowed to evolve collisionally over $2000 \square t$ with $\square_{0} \square t=0.001$; and the collision operator was applied every $10 \square t$. Kinetic energy and momentum conservation errors were $\sim 13 \%$ for NGP collisons and $\sim 3 \%$ for CIC compared to no collisions. Self-heating in particle $\operatorname{codes}^{27}$ and hybrid $\operatorname{codes}^{28}$ increases with decreasing $N_{\text {cell }}$ and was $\sim 38 \%$ with no collisions over $2000 \square \mathrm{t}$ in simulation bcoll17. We conclude that the errors in the NGP collisions are unacceptably large for $N_{\text {cell }}=16$.

The amount of self-heating in the absence of a laser field for $N_{\text {cell }}=16$ is significant. The SBS simulations in Ref. 2 used $N_{\text {cell }}=16$ for most of the results. However, self-heating does not compromise any of the physics conclusions in Ref. 2. At this point we examine self-heating issues. Figure 2 compares two collisionless simulations, bcoll17 with no incident laser field and bcem33n with an incident laser field. The 2D baseline simulation parameters in Ref. 2 are as follows: $6000 \square x \square 256 \square y$, $L_{\mathrm{x}}=192 \square_{\mathrm{o}}, L_{\mathrm{y}}=16 \square_{\mathrm{b}}, k_{0} \square x=0.2, \square_{\mathrm{pi}} \square t=0.2, \square_{\mathrm{s}} \square t=0.08, \square_{\mathrm{s}} \square=414 \sim 40 \mathrm{ps}, n_{\mathrm{e}} / n_{\mathrm{c}}=0.1, \mathrm{Be}$ plasma $(Z=4, A=9), \square_{\mathrm{e}}=\square x, m_{\mathrm{h}} / m_{\mathrm{e}}=1836, Z T_{\mathrm{e}} / T_{\mathrm{i}}=2$ to $16, T_{\mathrm{e}}=2 \mathrm{keV}$, collisionless, $\mathrm{v}_{0} / \mathrm{v}_{\mathrm{e}}=0.2\left(I_{0} \sim 2 \square 10^{15} \mathrm{~W} / \mathrm{cm}^{2}\right.$ for $\left.\square_{\mathrm{o}}=1 / 3 \square \mathrm{m}\right)$, number of ion superparticles $N_{\mathrm{i}}=10^{5} \square N_{\mathrm{y}}$ (2D), where $\square_{\mathrm{pi}}$ is the ion plasma frequency, $\square_{\mathrm{s}}$ is the ion acoustic frequency associated with the backscatter, $\square_{\mathrm{e}}$ is the electron Debye length, $\square_{0}$ is the incident laser wavelength, $T_{\mathrm{e}, \mathrm{i}}$ are the electron and ion temperatures, $L_{\mathrm{x}, \mathrm{y}}$ are the system dimensions, $\square x \approx \square y / 2$ are the 
grid sizes, $I_{0}$ is the laser intensity incident from the left, $n_{\mathrm{e}}$ is the electron density, $n_{\mathrm{c}}$ is the critical electron density, and $\square$ is the typical duration of the simulation. In these simulations $k_{\mathrm{y}}= \pm(0,1,2,3) \square 2 \square / L_{\mathrm{y}}$ for the electromagnetic wave field, and the full complement of 2D modes for the electrostatic potential was used. The simulations with this restriction on $k_{\mathrm{y}}$ for the electromagnetic wave field allow forward SBS, filamentation, and self-focusing at least in a limited way while preventing SBS sidescatter from being artificially preferred over backscatter because of the periodic boundaries in $y$. The results in Fig. 2 were obtained with $Z T_{\mathrm{e}} / T_{\mathrm{i}}=16$ and electron quiver velocity in the laser field $\mathrm{v}_{0} / \mathrm{v}_{\mathrm{e}}=0$ and 0.2 , respectively. We note that the self-heating effects are considerably smaller than the effects due wave-particle interactions accompanying strong SBS, which pull out a hot ion tail in the $x$ velocities and cause more transverse heating on the left side
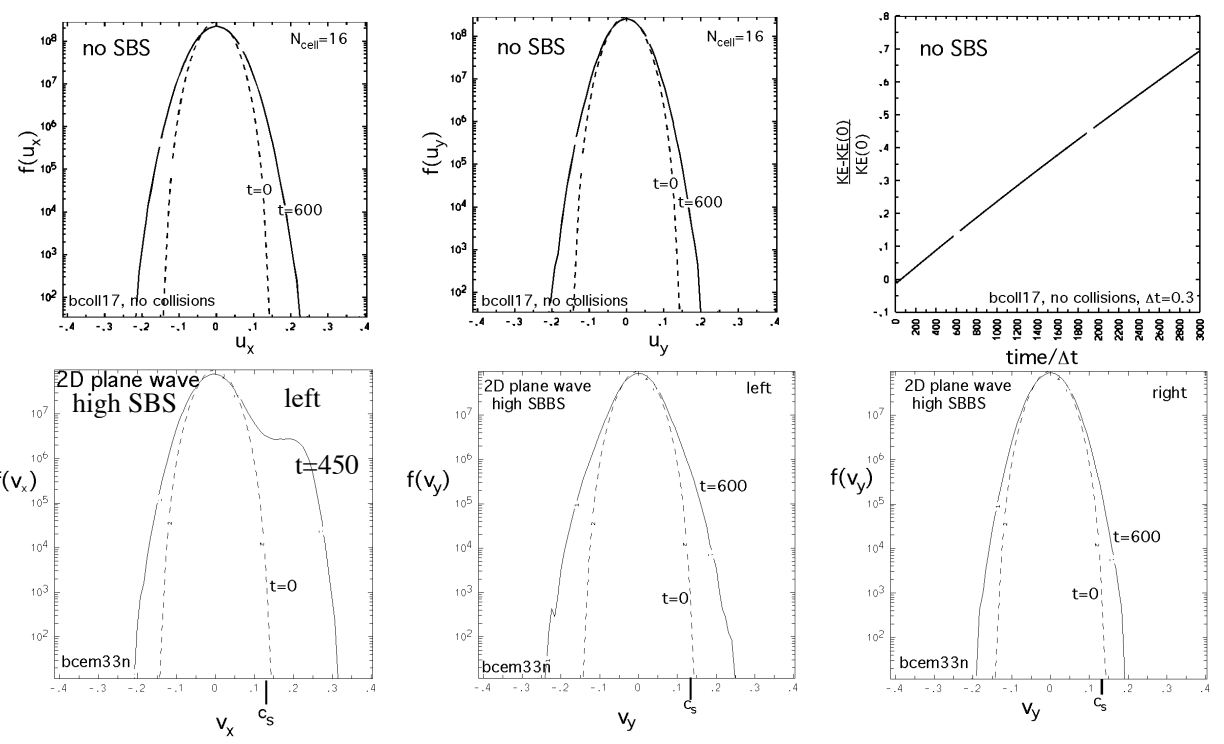

Figure 2. Snapshots of $x$ and $y$ ion velocity distribution functions from collisionless simulations with no incident laser (bcoll17) and with and incident laser and strong SBBS (bcem33n). Also shown is the relative growth of kinetic energy in bcoll17 vs. time step. 
of the $x$ domain where the SBBS ion wave and backscatter amplitudes are largest than on the right side of the domain farther into the plasma.

Birdsall and Langdon ${ }^{27}$ point out that smoothing of the charge density to partially suppress short wavelength fluctuations before solving for the self-consistent electric potential reduces the self-heating rate. The self-heating rate for $Z T_{\mathrm{e}} / T_{\mathrm{i}}=16$ was reduced three-fold from $2.4 \square 10^{-4} / \square t$ in bcoll17 to $0.8 \square 10^{-4} / \square t$ in bcoll18 with local digital smoothing $^{27}$ of the ion charge density using a five-point stencil, $(1,1,4,1,1) / 8$. The fivepoint stencil produces a multiplicative smoothing factor in $\left(k_{\mathrm{x}}, k_{\mathrm{y}}\right)$ space: $(1 / 2)\left(\cos ^{2}\left(k_{x} \square x / 2\right)+\cos ^{2}\left(k_{y} \square y / 2\right)\right)$. Figure 3 shows results from bcoll18, which can be compared directly to bcoll17 results in Fig. 2. We obtain even better smoothing results using successive 1D three-point stencils, $(1,2,1) / 4$, first in $x$ and then in $y$, for which the $\boldsymbol{k}$-space smoothing factor is $\cos ^{2}\left(k_{x} \square x / 2\right) \cos ^{2}\left(k_{y} \square y / 2\right)$; and the observed heating rate is further reduced to $0.2 \square 10^{-4} / \square$ t for the same parameters as in bcoll17 and bcoll18. In using digital smoothing in SBBS simulations when no deliberate seeding of the backscatter electromagnetic wave is introduced, one needs to be mindful that the smoothing reduces the thermal noise in the charge densities, which then beat with the incident laser to provide a reduced noise source for the backscatter. The reduced noise source can then affect the reflectivity. Figure 4 shows an SBBS simulation using the baseline parameters with $Z T_{\mathrm{e}} / T_{\mathrm{i}}=16$ and five-point stencil smoothing of the ion charge density. These results can be compared directly to the bcem $33 \mathrm{n}$ results (no smoothing) shown in Fig. 2. Production of a hot ion tail in $x$ and transverse heating in $y$, both of which are more pronounced on the left side of the domain where the backscatter and IAW amplitudes are strongest, is more obvious with the application of digital smoothing. 

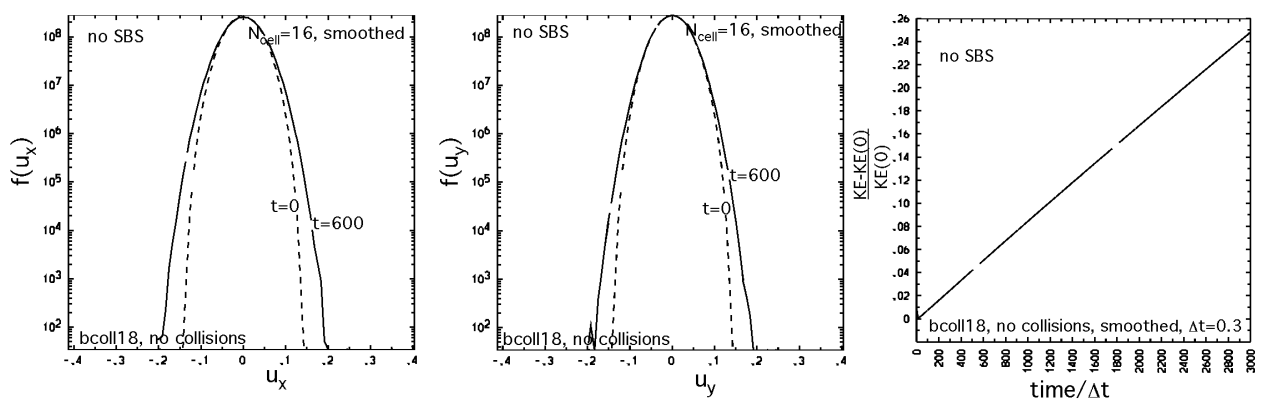

Figure 3. Collisionless 2D simulation bcoll18 with no incident laser and with digital smoothing of the ion charge density: snapshots of the $x$ and $y$ ion velocity distribution functions and relative ion kinetic energy growth vs. time step.
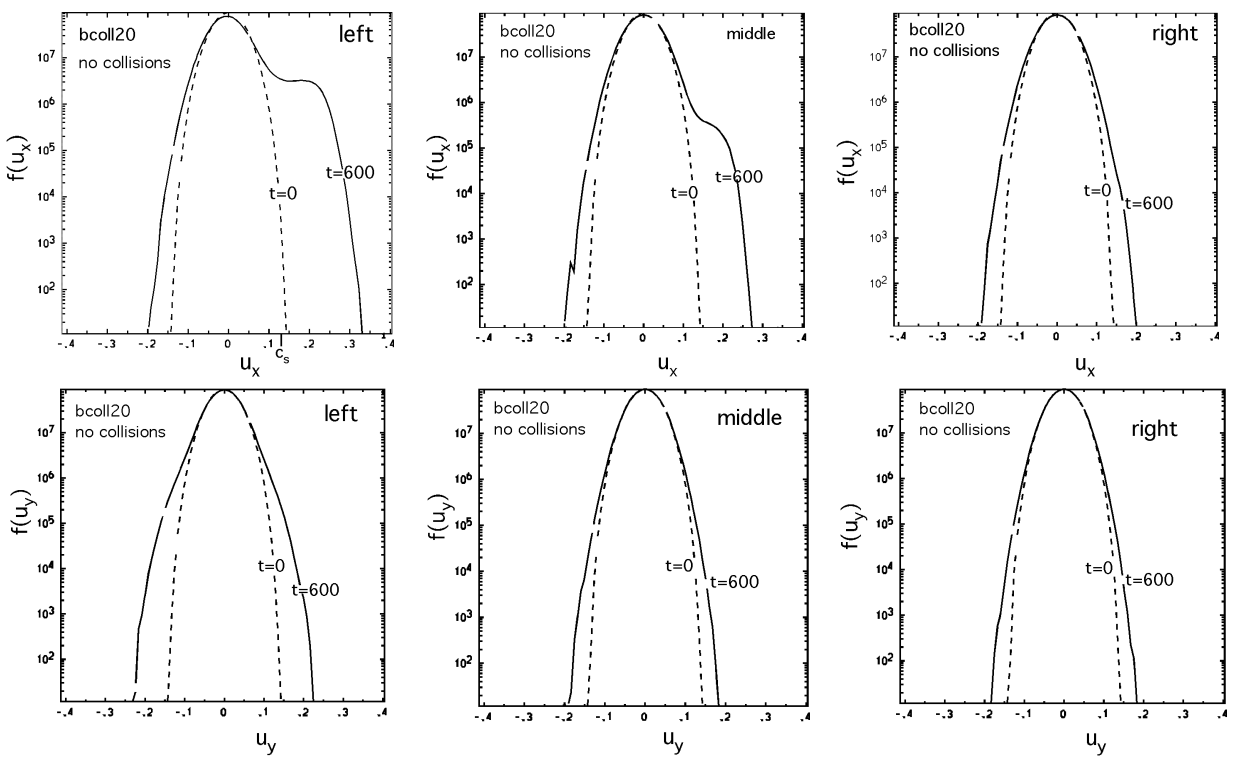

Figure 4. Collisionless 2D simulation bcoll20 with five-point-stencil digital smoothing of the ion charge density and strong SBBS: snapshots of the $x$ and $y$ ion velocity distribution functions over regions centered at $x=L_{\mathrm{x}} / 4, L_{\mathrm{x}} / 2$, and $3 L_{\mathrm{x}} / 4$ and integrated in $y$.

We now return to consideration of test simulations for the collision algorithm of Jones, et al. The relaxation of a strong temperature anisotropy is a convenient test case. A 2D anisotropic Maxwellian $f_{\mathrm{i}}\left(\mathrm{u}_{\mathrm{x}}, \mathrm{u}_{\mathrm{y}}\right)$ was initialized with $T_{\mathrm{y}}=0$ and $T_{\mathrm{x}} \neq 0$ in bcoll23. The plasma was allowed to evolve collisionally over $1000 \square t$ with $N_{\text {cell }}=16$ and $\square \square \square t=0.001$ The 
collision operator was applied every $10 \square$ t. The simulation results in Fig. 5 agree fairly well with the analytical solution for the relaxation of the temperature difference, which is given by $T_{x} \square T_{y}=\left(T_{x 0} \square T_{y o}\right) \exp \left(\square 2 \square_{0} t\right)$ for a collision operator with no velocity dependence. We undertook an additional test simulation with the Jones, et al. collision algorithm, the relaxation to a Maxwellian of an initially square velocity distribution function. The relaxation is quite rapid as reported in Ref. 23 . We return to this test case in the next sub-section.
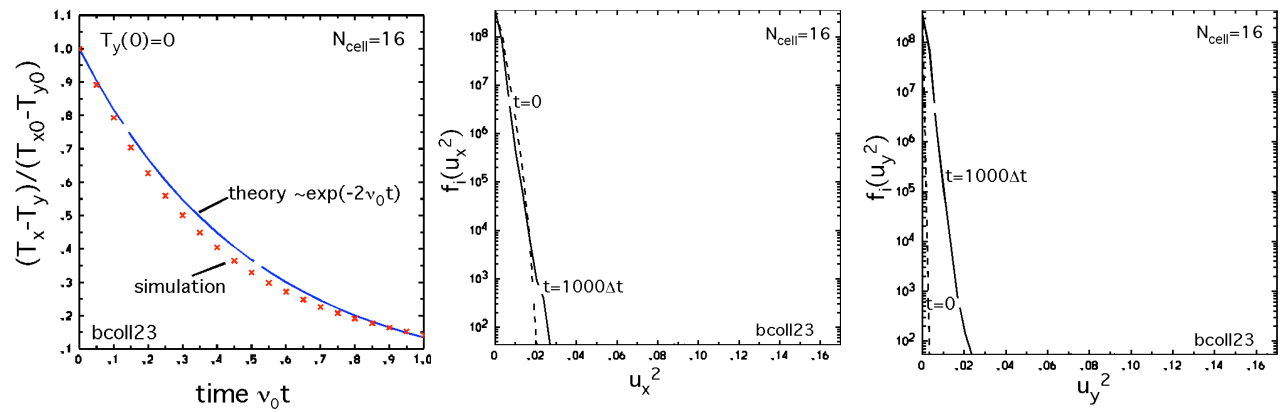

Figure 5. Relaxation of a strong temperature anisotropy: relative anisotropy vs. time and snapshots of the ion velocity distribution $\left(\log _{10}(f)\right.$ vs. square of velocities, $u_{\mathrm{x}}{ }^{2}$ and $\left.u_{\mathrm{y}}{ }^{2}\right)$.

\section{Fokker-Planck Collision Operator}

The second Coulomb collison operator that we implemented retains the proper velocity dependence of a Fokker-Planck Coulomb collision model. ${ }^{24,25}$ The Jones et al. collision operator has no velocity dependence in the drag and diffusion coefficients.. References 24 and 25 present Monte Carlo collision algorithms (Langevin representation) with drag and diffusion tensor coefficients $\left\langle\square W_{\|}\right\rangle,\left\langle\square W_{\|}^{2}\right\rangle,\left\langle\square W_{\square}^{2}\right\rangle$ per $\square$ t for the velocity drag parallel $(<0)$ to the test particle velocity vector and the variances for the diffusive increments for parallel and perpendicular velocities that are functions of test particle speed and can be calculated in terms of error functions for an assumed isotropic 
Maxwellian target distribution on which the test particles scatter. The diffusive kicks satisfy Gaussian statistics in Ref. 25. In the reference frame co-moving with the local mean drift, the $3 \mathrm{D}$ collision operator represented as a Langevin equation to $O(\square \mathrm{t})$ is

$$
\square \mathbf{v}=\mathbf{F}_{d} \square t+\mathbf{Q}
$$

Reference 25 outlines how to represent the fully nonlinear Fokker-Planck equation for Coulomb collisions within a particle code by solving for the two scalar Rosenbluth potentials from which $\mathbf{F}_{\mathrm{d}}$ and $\mathbf{Q}$ are self-consistently determined, which involves integrating over the target-particle velocity distribution function as a function of the test-particle velocity. Here we approximate the target velocity distribution as an isotropic Maxwellian using the evolving local ion density and temperature, for which the Langevin Fokker-Planck collision operators introduced in Refs. 24 and 25 are fully equivalent. We refer the reader to Refs. 24 and 25 for specific formulae used in the collision operator. Of particular interest is the discussion in Ref. 25 of the differences in the drag and diffusion coefficients between the Fokker-Planck collision operator and the Jones, et al. collision operator, which are graphed in Fig. 1 of Ref. 25. The Jones, et al. collision operator is relatively less collisional at low energies and much more collisional at high energies. We make use of two features in the algorithm introduced in Eqs. (20) and (24) of Ref. 25 that improve energy conservation by compensating for errors due to the expansion in $\square * \square t$ inherent in the Langevin equation and due to the statistical nature of the collision algorithm:

$$
F_{d} \square F_{d}\left(1 \square F_{d} \square t / 2 \mathrm{v}\right), F_{d}<0
$$

where $\mathrm{v}$ is the test-particle speed in the local mean-drift frame and $\left|F_{d} \square t / \mathrm{v}\right|<1$ is required for the expansion to be sensible; and after the collisional scattering occurs in the 
mean-drift frame, the particle velocities are renormalized by a factor that is very close to unity,

$$
\mathbf{v}^{\prime \prime}=\left(K E_{j} / K E_{j}\right)^{1 / 2} \mathbf{v}^{\prime}
$$

where $K E_{\mathrm{j}}$ is the kinetic energy in the mean drift frame of the $j$ th block of particles (the block local to the test particle) before the collisional scattering and $K E_{\mathrm{j}}{ }^{\prime}$ is the corresponding kinetic energy after the collisional scattering.

To streamline the computation of the drag and diffusion coefficients in the Fokker-Planck collision operator, we introduce simple Padé approximations that are fit with a least-squares determination relative to the error functions in Refs. 24 and 25:

$$
\begin{aligned}
& F_{d} \square t / v=\left\langle\square W_{\|}\right\rangle / \mathbf{v}=\square \square \square t /\left(1+1.08\left(\mathbf{v} / \sqrt{2} \mathbf{v}_{t h}\right)^{2.46}\right) \\
& \left\langle\square W_{\|}^{2}\right\rangle / \mathbf{v}_{t h}^{2}=\square\left\langle\square W_{\|}\right\rangle / \mathbf{v} \\
& \left\langle\square W_{\square}^{2}\right\rangle / \mathbf{v}_{t h}^{2}=\square_{\square} \square t /\left(1+0.186\left(\mathbf{v} / \sqrt{2} \mathbf{v}_{t h}\right)^{1.85}\right)
\end{aligned}
$$

where $\square *=2^{3 / 2} \square_{0}$. The Padé approximants are compared to the numerical evaluations of the drag-diffusion coefficients computed from the error functions in Fig. 6. The random velocity vector $\left.\mathbf{Q}=\left(Q_{\|}, Q_{\prod 1}, Q\right\rceil 2\right)$ for velocity displacements parallel and perpendicular to the test-particle velocity vector in the local mean-drift frame is chosen from the Gaussian probability distribution function:

$$
\square(\mathbf{Q})=\frac{1}{(2 \square)^{3 / 2}<W_{\|}^{2}>^{1 / 2}<W_{\square}^{2}>} \exp \stackrel{\square}{\square} \frac{Q_{\|}^{2}}{2<W_{\|}^{2}>} \square \frac{Q_{\prod 1}^{2}+Q_{\prod 2}^{2}}{2<W_{\square}^{2}>}
$$

This Langevin Fokker-Planck collision operator is only consistent with a threedimensional velocity representation; therefore we define a third velocity coordinate $\mathrm{v}_{\mathrm{z}}$ in BZOHAR that does not respond to electric fields and only participates in the collisions. 

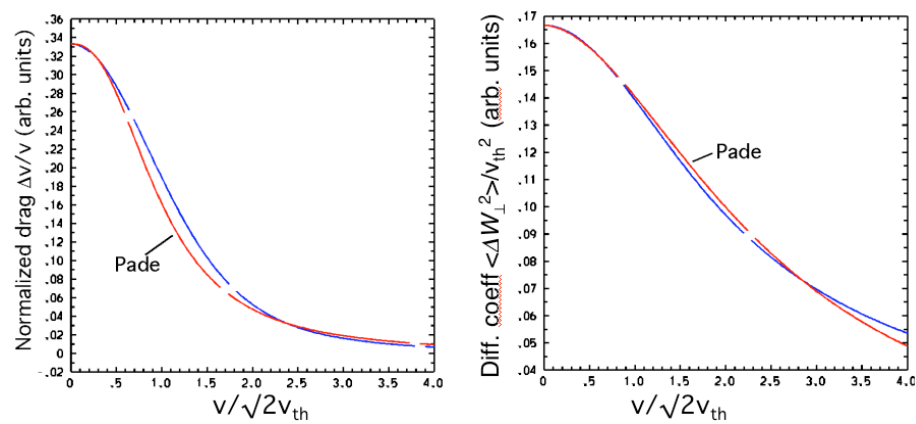

Figure 6. Padé approximations for the Fokker-Planck drag and perpendicular velocity variance diffusion coefficients vs. $\mathrm{v} /\left(2 \mathrm{v}_{\mathrm{th}}{ }^{2}\right)^{1 / 2}$ compared to those calculated numerically from error functions.

The first test case with the Fokker-Planck collision operator was preserving a Maxwellian ion velocity distribution with no laser fields present. In Fig. 7 we show the results of collisionally evolving the ion velocity distribution functions using CIC interpolation, $N_{\text {cell }}=16$, $\square \square t=0.001$, collisions applied every $10 \square t$, and with five-pointstencil local smoothing of the ion charge density. The 3D Maxwellian is preserved in bncoll3, and the relative self-heating rate is $0.35 \times 10^{-4} / \square t$. The 3D Fokker-Planck collision algorithm is 2-3 times slower than the Jones, et al. 2D collision algorithm.
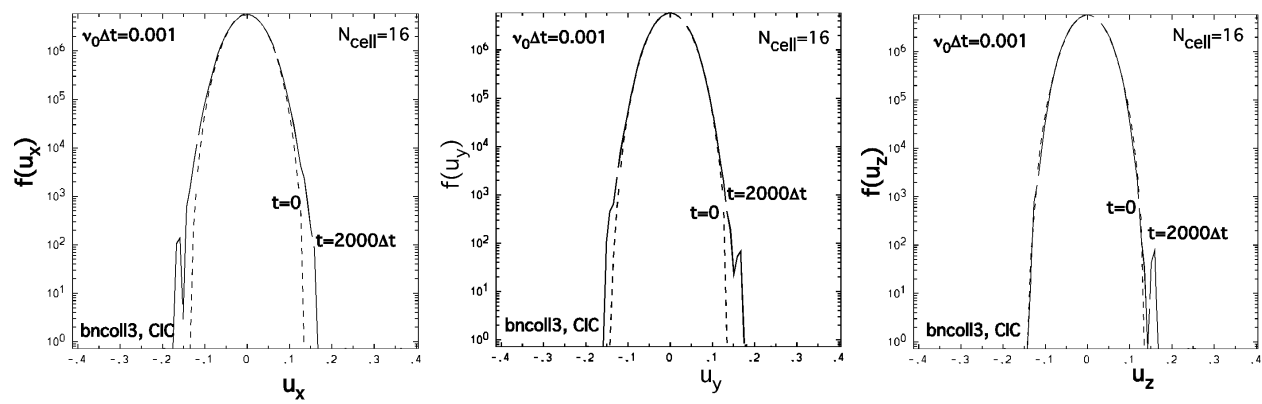

Figure 7. Snapshots of the 3D ion velocity distribution function at $t=0$ and $2000 \square t$ with $\square_{0} \square t=0.001$ and Fokker-Planck collisions preserving a Maxwellian (simulation bncoll3).

The second test case with the Fokker-Planck collision operator was the relaxation 
of a weak temperature anisotropy. A 3D anisotropic Maxwellian $f_{\mathrm{i}}\left(\mathrm{u}_{\mathrm{x}}, \mathrm{u}_{\mathrm{y}}, \mathrm{u}_{\mathrm{z}}\right)$ was initialized with $T_{\mathrm{y}}=0.9 T_{\mathrm{x}}$ and $T_{\mathrm{x}}=T_{\mathrm{z}} \neq 0$. The plasma was allowed to evolve collisionally over $1000 \square t$ with $N_{\text {cell }}=16$ and $\square_{0} \square t=0.0005$ The collision operator was applied every $7 \square t$ with $\square \square t_{\mathrm{c}}=0.01$ The simulation results (bncoll7c) are compared in Fig. 8 with an approximate analytical solution for the relaxation of a weak temperature anisotropy, which is given by Trubnikov ${ }^{29}: T_{z} \square T_{y}=\left(T_{z 0} \square T_{y o}\right) \exp \left(\square 0.23 \square_{0}^{i i} t\right)$, where $\square_{o}^{i i}=3 \square^{1 / 2} \square_{0}$. With a stronger relative temperature anisotropy at $t=0$, a faster relaxation rate is observed, as is expected from theory.

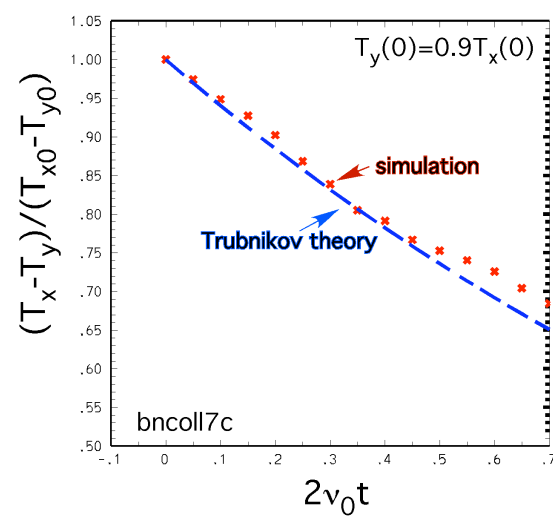

Figure 8. Fokker-Planck collisional relaxation of a weak temperature anisotropy (initially $T_{\mathrm{y}}=0.9 T_{\mathrm{x}}$ and $\left.T_{\mathrm{x}}=T_{\mathrm{z}} \neq 0\right)$ with $N_{\text {cell }}=16$ and $\square_{\mathrm{b}} \square \mathrm{t}=0.0005$ : relative temperature anisotropy vs. dimensionless time $2 \square_{0} t$.

A third test case of the Fokker-Planck collision operator was undertaken in which a 3D isotropic, square velocity distribution $f_{\mathrm{i}}\left(u_{\mathrm{x}}, u_{\mathrm{y}}, u_{\mathrm{z}}\right)=$ const for $\left|u_{\mathrm{x}}\right|,\left|u_{\mathrm{y}}\right|,\left|u_{\mathrm{z}}\right|<\sqrt{3} \mathrm{v}_{\mathrm{i}}$ was initialized. The plasma was allowed to collisionally relax with $N_{\text {cell }}=16$ and $\square \square \square t=0.5 \square 10^{-3}$ Fokker-Planck collisions were applied every 7 $\square \mathrm{t}$ with $\square: \square t_{\mathrm{c}}=0.01 f_{\mathrm{i}}\left(\mathrm{u}_{\mathrm{x}}, \mathrm{u}_{\mathrm{y}}, \mathrm{u}_{\mathrm{z}}\right)$ rapidly relaxes toward a Maxwellian. Snapshots of the collisionally evolving velocity 
distribution functions for the velocity-independent and Fokker-Planck collision schemes are presented in Fig. 9. Formation of the Maxwellian tail is faster with the velocityindependent collisions.

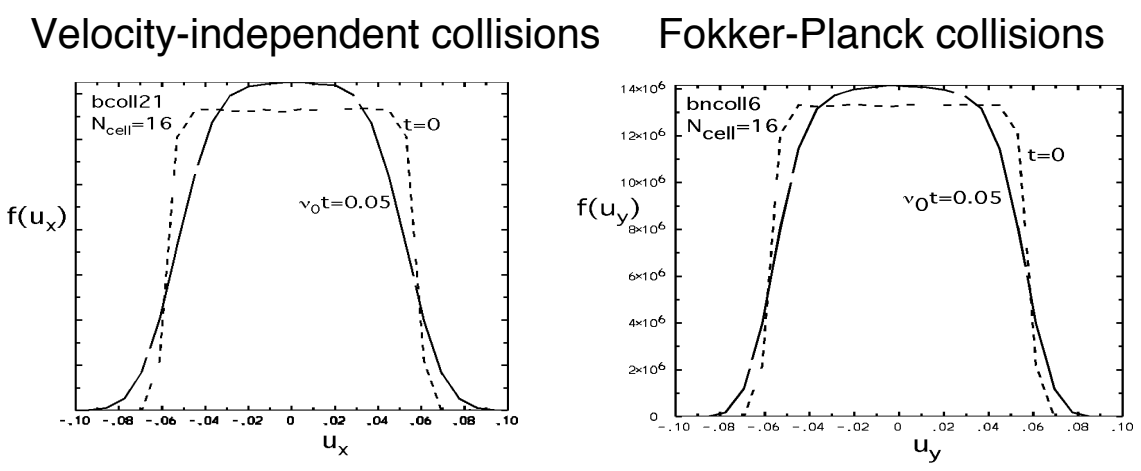

Figure 9. Relaxation of an initially square velocity distribution function. Snapshots of ion velocity distribution functions from simulations with velocity-independent collisions (bcoll21) and Fokker-Planck collisions (bncoll6), $N_{\text {cell }}=16$ and $\square \square \square t=0.5 \square 10^{-3}$

As a final test case for the Fokker-Planck collision operator, we examined the damping of a small-amplitude ion acoustic wave for finite collisionality. Both ion Landau damping and ion collisions contribute to the damping rate. ${ }^{30} \mathrm{We}$ initialized a standing wave with $Z T_{\mathrm{e}} / T_{\mathrm{i}}=8, k=0.052, \square_{\mathrm{e}}=\square x=0.2, \square t=0.3, N / \square x \square y=64, k_{\mathrm{y}}=0$, and $\square_{0} \square t=8 \times 10^{-4}$ (bcoll30f) or collisionless (bcoll30e). Collisionless theory gives $\square+i \square=0.0085 \square i 0.00026$, while in the simulation (bcoll30e) $\square+i \square=0.0082 \square i 0.00026$ is observed (Fig. 10). With collisions and $k \ell_{i}=k v_{i} / \sqrt{2} Z_{0}=4.4$, collisional linear theory ${ }^{30}$ gives $\square+i \square=0.0084 \square i 0.00032$, while in the simulation (bcoll30f) $\square+i \square=0.0081 \square i 0.00029$ is observed (Fig. 10). We note that $[n / n=0.05$ initially. At this wave amplitude, the ion trapping frequency is $\square_{\text {trap }} / \operatorname{Re} \square \sim \mid\left[n_{e} /\left.n_{e}\right|^{1 / 2} \sim 0.2\right.$, so that $2 \square \square$ trap 3400 , from which we conclude that trapping effects (negative frequency 
shift and damping reduction) should set in for $\mathrm{t}>1700$ in the simulations. ${ }^{31}$ In consequence, data for the frequency and damping rate should be compared to linear theory over just the first two periods of the wave oscillation. Agreement of the simulations (Fig. 10) with linear theory is within $10 \%$.
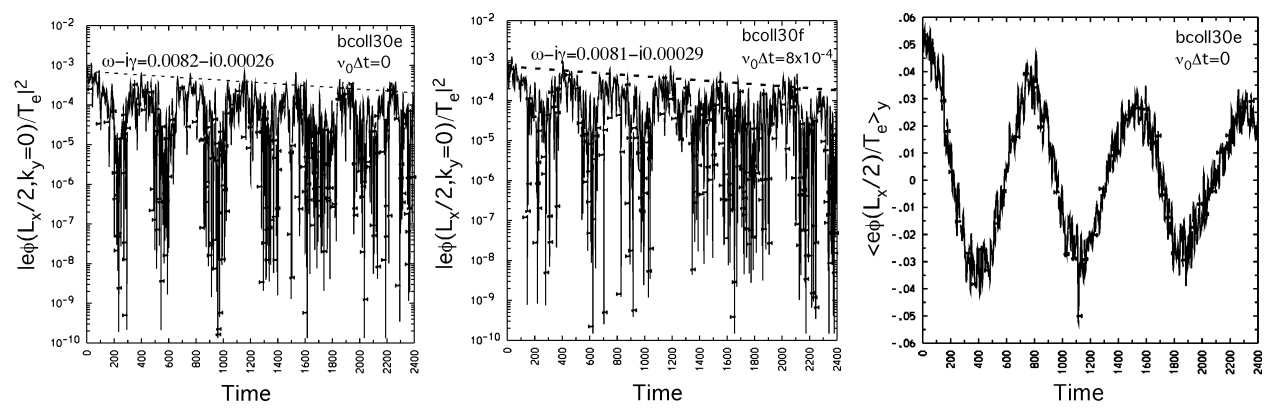

Figure 10. Damping of an ion acoustic wave due to ion Landau damping and collisions for a collisionless simulation (bcoll30e) and a collisional simulation (bcoll30f, $\left.\square \square t=8 \times 10^{-4}, k \ell_{i}=4.4\right)$ : mode amplitudes as functions of time at $x=L_{\mathrm{x}} / 2$.

\section{STIMULATED BRILLOUIN SCATTERING WITH ION COLLISIONS}

We have performed simulations studying stimulated Brillouin backscattering in two spatial dimensions including ion-ion collisions varying the relative collisionality and using the two different collision algorithms. We first estimate the relative collisionality for the conditions corresponding to our baseline parameters introduced in Ref. 2 and used here. For an incident laser with wavelength $\square_{0}=1 / 3 \square \mathrm{m}$, electron density $n_{\mathrm{e}} \sim 10^{21} \mathrm{~cm}^{-3}$, $n_{\mathrm{e}} / n_{\mathrm{c}} \sim 0.1$ relative to critical density, and a beryllium plasma with electron temperature $T_{\mathrm{e}}=2 \mathrm{keV}$ and $Z T_{\mathrm{e}} / T_{\mathrm{i}}=16$, the characteristic ion-ion collision frequency is $\square_{0}^{i i}=3 \square^{1 / 2} \square_{0} \sim 4 \square 10^{11_{s} \square 1}$ and $\square 0 \sim 0.8 \square 10^{11}{ }_{s} \square 1_{\text {using Eq.(2). This can be compared }}$ to the ion acoustic frequency $\square_{\mathrm{s}} \sim 2 \square 10^{13} \mathrm{rad} / \mathrm{s}$ in such a plasma for backscatter with $k \rrbracket_{\mathrm{e}}=0.4$, and the trapped ion bounce frequency for a wave amplitude $I\left[n_{\mathrm{e}} / n_{\mathrm{e}} \mid \sim 0.01\right.$ is 
$\square_{\text {trap }}=\left(\left[\mathrm{h}_{e} / \mathrm{n}_{e}\right)^{1 / 2} \square_{s} \sim 2 \square 10^{12} \mathrm{rad} / \mathrm{s}\right.$. Thus, two orders of magnitude separate the ion collision frequency and the ion acoustic frequency; and ions can be trapped before they are collisionally decorrelated from the ion wave if the ion wave is sufficiently large. The

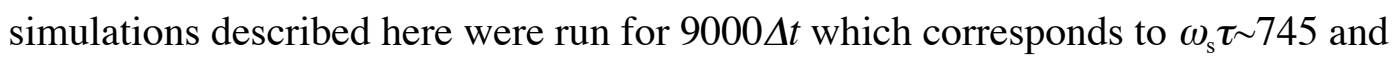
$\sim 70 \mathrm{ps}$; and the incident laser was uniform in $y$ at $x=0$.

Figure 11 shows the results of a series of simulations using the Jones, et al., collision algorithm for $Z T_{\mathrm{e}} / T_{\mathrm{i}}=16$ and our baseline parameters. Instantaneous and average SBBS reflectivities decrease with increasing collisionality. Local smoothing of the charge density with successive three-point, one-dimensional smoothing in $x$ and $y$ was used. The amplitude gain exponent in Fig. 11 is calculated from ${ }^{8,11,32}$

$$
G_{S B B S}^{a}=\frac{1}{16} \frac{\mathrm{v}_{0}^{2}}{\mathrm{v}_{e}^{2}} \frac{n_{e}}{n_{c}} \frac{\square_{s}}{\square_{s}} \frac{\square_{0} L_{x}}{v_{g}\left(1+k^{2} \square_{e}^{2}\right)}
$$

where $\square$ is the ion wave damping rate, $\square_{0}$ is the pump laser frequency, $\mathrm{v}_{\mathrm{g}}$ is the laser group velocity, and $k$ is the SBBS ion wave wavenumber. For the velocity-independent collision operator, the total ion-wave linear damping rate is given approximately by $\square_{s}=\square_{L D}+\square_{0} / 2$, where $\square_{\mathrm{D}} \sim 0.0023 \square_{\mathrm{s}}$ is the ion Landau damping rate for $Z T_{\mathrm{e}} / T_{\mathrm{i}}=16$. Figures 12 and 13 show results from one of the simulations (bcoll26, $\square \square t=2 \square 10^{-4}$ ) from the series in Fig. 11. The saturation of SBBS is qualitatively similar to the collisionless results analyzed in Ref. 2: ion trapping and secondary instability of the SBBS ion wave accompany a temporal crash of the SBBS reflectivity, and late in time forward Brillouin scattering becomes pronounced. The forward Brillouin scattered light is identified in the power spectrum of the transmitted light, which has features that are frequency downshifted slightly from the incident light frequency; moreover, the system is not wide 
enough in $y$ to support filamentation. ${ }^{2}$ Backscatter is dominant on the left, and ion trapping produces an energetic tail. Nonlinear IAW decay has heated the transverse velocity distribution at the time shown while collisions isotropize the distribution function over a longer time.
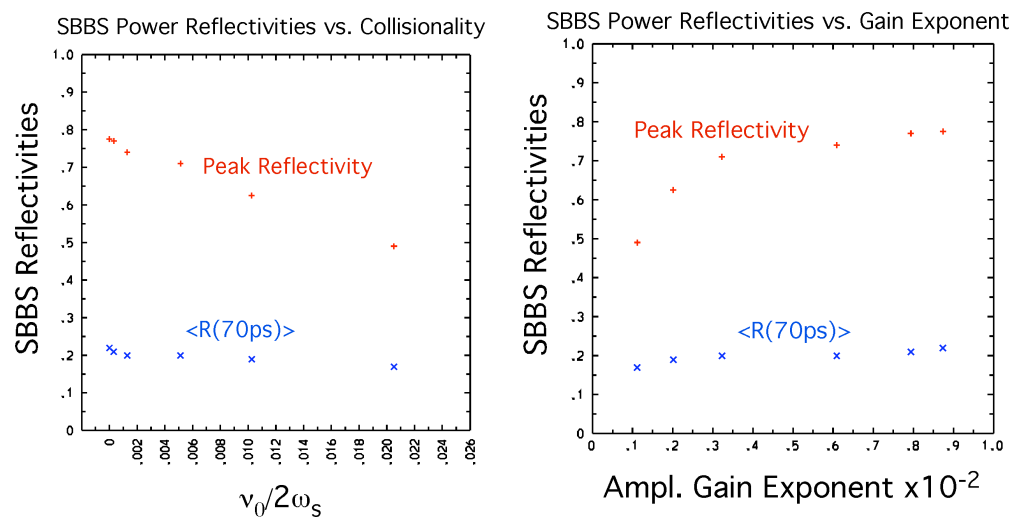

Figure 11. Peak and 70ps average SBBS reflectivities for parameters: $Z T_{\mathrm{e}} / T_{\mathrm{i}}=16$, $\mathrm{v}_{0} / \mathrm{v}_{\mathrm{e}}=0.2, k_{0} \square_{\mathrm{e}}=0.2, m_{\mathrm{Be}} / m_{\mathrm{e}}=$ real mass ratio, $n_{\mathrm{e}} / n_{\mathrm{c}}=0.1$, and Jones, et al. collision model
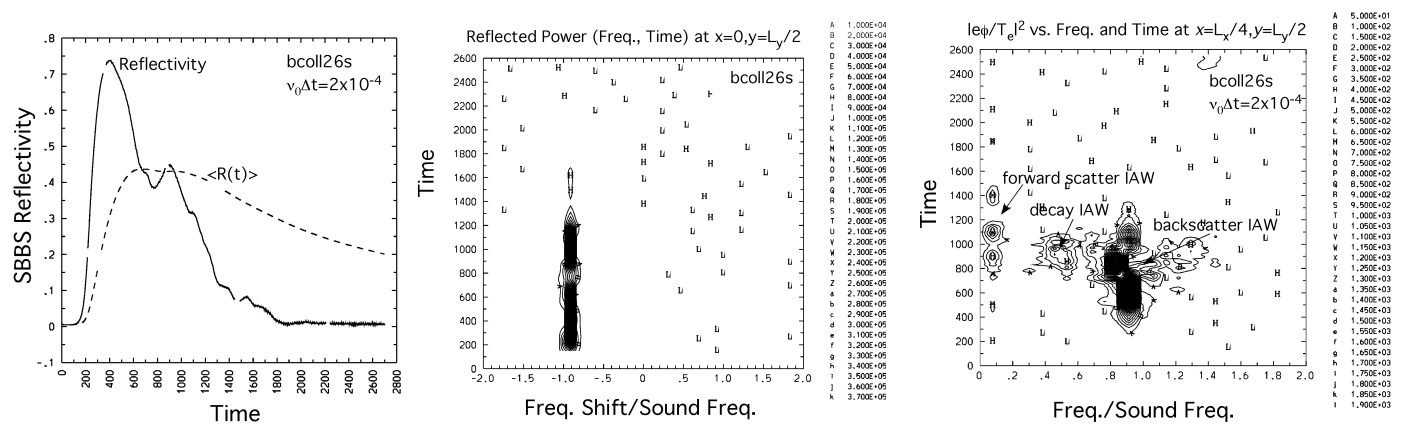

Figure12. SBS simulation bcoll26 with velocity-independent collisions and parameters: $Z T_{\mathrm{e}} / T_{\mathrm{i}}=16, \mathrm{v}_{0} / \mathrm{v}_{\mathrm{e}}=0.2, k_{0} \square_{\mathrm{e}}=0.2$, real mass ratio for $\mathrm{Be}, n_{\mathrm{e}} / n_{\mathrm{c}}=0.1$, and $\square \mathrm{b} \square t=2 \square 10^{-4}$ Instantaneous and average reflectivity, $R(t)$ and $<R(t)>=t^{\square}{ }^{t} \square d t^{\prime} R\left(t^{\prime}\right)$; reflected electromagnetic power spectrum vs. time at $x=0$ and $y=L_{\mathrm{y}} / 2$ vs. $\left(\square \square \square_{0}\right) / \square_{\mathrm{s}}$ and time; power spectrum for $\mid e \square\left(L_{x} / 4, L_{y} /\left.2\right|^{2}\right.$ vs. $\square / \square_{\mathrm{s}}$ and time.
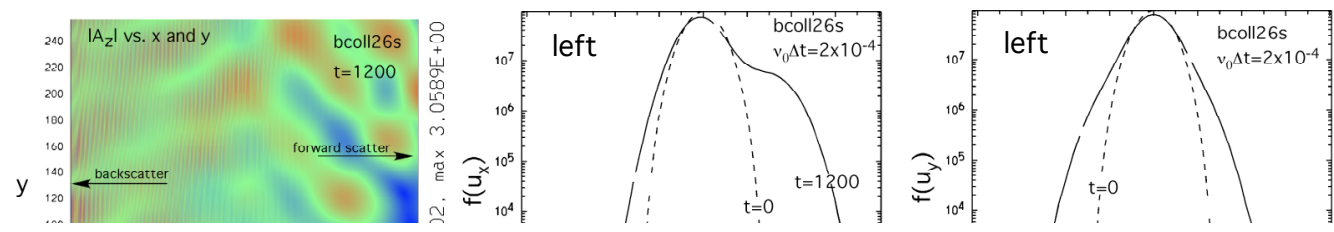
Figure13. SBS simulation bcoll26 with velocity-independent collisions and parameters: $Z T_{\mathrm{e}} / T_{\mathrm{i}}=16, \mathrm{v}_{0} / \mathrm{v}_{\mathrm{e}}=0.2, k_{0} \square_{\mathrm{e}}=0.2$, real mass ratio for $\mathrm{Be}, n_{\mathrm{e}} / n_{\mathrm{c}}=0.1$, and $\square \square \square t=2 \square 10^{-4}$ Electromagnetic potential $\left|A_{\mathrm{z}}\right|$ vs. $x$ and $y$ at $\mathrm{t}=1050 ; x$ and $y$ velocity distribution functions on the left side of the domain at $t=0$ and 1200.

A series of two-dimensional BZOHAR simulations was performed with FokkerPlanck collisions over a range of collisionality: $\square \square \square t=\left\{5 \square 10^{-5}, 2 \square 10^{-4}, 8 \square 10^{-4}, 1.6 \square 10^{-3}\right\}$. Figure 14 shows the results of the SBBS reflectivity as a function of collisionality parameter and the concomitant linear convective gain using Eq. (10) and the FokkerPlanck calculations due to Randall ${ }^{30}$ for the linear ion-wave damping rate due to ion Landau damping and ion-ion collisions. The baseline simulation parameters introduced earlier for $Z T_{\mathrm{e}} / T_{\mathrm{i}}=16$ were used. We note that the normalized Fokker-Planck drag and parallel diffusion coefficients for a Maxwellian velocity distribution in Ref. 25 [approximated in Eqs.(8a) and (8b) here] are significantly smaller than the corresponding value in the Jones, et al., velocity-independent collision model at the ion velocity resonant with the SBBS ion wave: $\mathrm{v}_{\mathrm{x}} / \mathrm{v}_{\mathrm{i}}=\left(Z T_{\mathrm{e}} / T_{\mathrm{i}}\right)^{1 / 2}=4$, so that the resonant ions are less collisional in the Fokker-Planck mode. Moreover, the ion wave damping rate $\square$ with Fokker-Planck collisions is $\mathrm{O}(1 / 3)$ of that in the velocity-independent collision model for $\square_{0}>>\square_{\mathrm{D}}$ and $Z T_{\mathrm{e}} / T_{\mathrm{i}}=16$ (compare Table I in Ref. 30 to $\square_{s}=\square_{L D}+\square_{0} / 2$ for the velocityindependent collisions). The peak SBBS reflectivity decreases very weakly with increasing collisionality, while the average reflectivity over $70 \mathrm{ps}$ increases significantly. 
The values of the peak reflectivities with a small decrease over the entire range of collisionality are similar to the reflectivities over the reduced range of collisionality $0 \square \square \square t \square 4 \square 10^{-4}$ in the simulations with the velocity-independent collision model to account for the difference in the relative collisionalities of the resonant ions between the two collision models. The increase of the average reflectivity with increasing collisionality is due to the crash of the SBBS proceeding more slowly with increased collisionality and Fokker-Planck collisions. We note that the threshold for the two-ion wave decay increases with increasing ion collisionality and its growth rate above threshold decreases. Could collisional inhibition of the two-ion-wave decay account for the slower rate of crash of the SBBS reflectivity? However, the crash times and averaged reflectivies do not increase with increasing collisionality in our SBBS
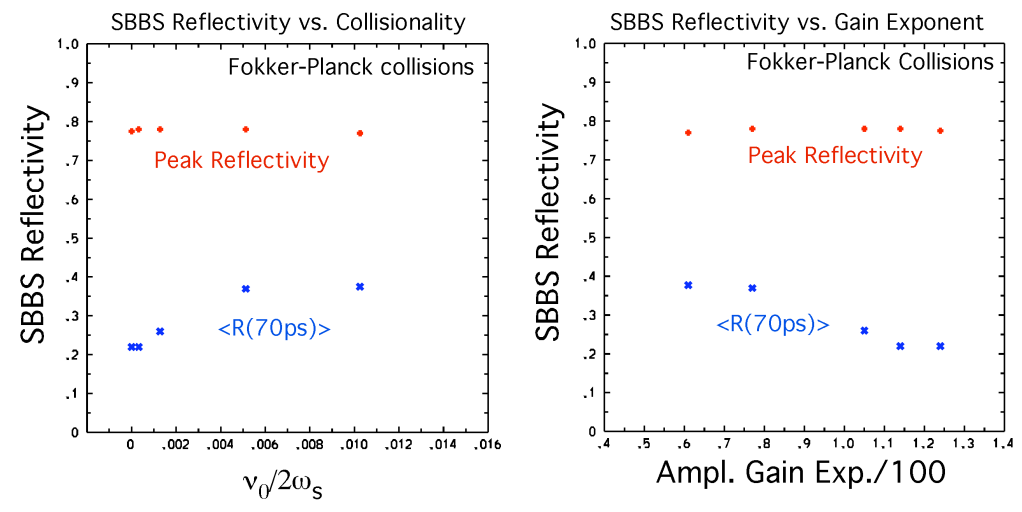

Figure 14. Peak and average SBBS reflectivities as functions of collisionality (FokkerPlanck collisions) and corresponding linear gain exponent for parameters: $Z T_{\mathrm{e}} / T_{\mathrm{i}}=16$, $\mathrm{v}_{\mathrm{o}} / \mathrm{v}_{\mathrm{e}}=0.2, k_{0} \square_{\mathrm{e}}=0.2, m_{\mathrm{Be}} / m_{\mathrm{e}}=$ real mass ratio, $n_{\mathrm{e}} / n_{\mathrm{c}}=0.1, L_{\mathrm{x}} \sim 192 \square$, and $L_{\mathrm{y}} \sim 16 \square$. 
simulations with the velocity-independent collision model. Examination of the simulations in detail leads to a possible explanation for this difference in the simulation results from the two collision models.

Figures 15 and 16 show results from a simulation (bcoll26nn) in which FokkerPlanck collisions were used with $\square\left[\square t=2 \square 10^{-4}\right.$ and our baseline parameters. The results here should be compared to those in Figs. 12 and 13 for the same parameters, but in which the velocity-independent collision model was used. The qualitative features are quite similar: ion trapping and ion wave decay are observed; a hot ion tail is formed in the longitudinal velocity distribution preferentially near the entrance to the plasma of the incident laser where the SBBS ion wave amplitude is large; and there is transverse ion heating where the SBBS ion wave is large. Collisions isotropize the velocity distribution function and return it to a Maxwellian over a longer time. After the SBBS reflectivity
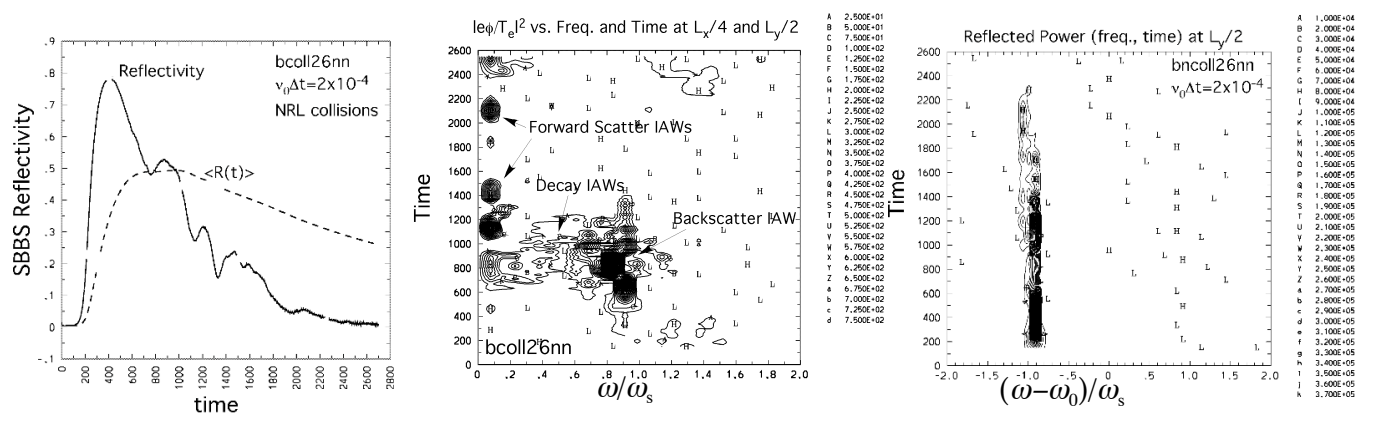

Figure 15. SBBS instantaneous and cumulative time-average reflectivities vs. time, power spectrum for $\mathrm{l} e\left[\left(L_{\mathrm{x}} / 4, L_{\mathrm{y}} / 2\right) /\left.T_{\mathrm{e}}\right|^{2} \mathrm{vs}\right.$. $\square / \square_{\mathrm{s}}$ and time, and power spectrum for reflected electromagnetic power vs. $\left(\square \square \square_{0}\right) / \square_{\mathrm{s}}$ and time, for parameters: $Z T_{\mathrm{e}} / T_{\mathrm{i}}=16, \mathrm{v}_{0} / \mathrm{v}_{\mathrm{e}}=0.2$, $k_{0} \square_{\mathrm{e}}=0.2, m_{\mathrm{Be}} / m_{\mathrm{e}}=$ real mass ratio, $n_{\mathrm{e}} / n_{\mathrm{c}}=0.1, L_{\mathrm{x}} \sim 192 \square_{0}, L_{\mathrm{y}} \sim 16 \square_{b}$, Fokker-Planck collisions, and $\square\left[\square t=2 \square 10^{-4}\right.$ (bcoll26nn).

crashes, forward SBS is observed. The SBBS reflectivity with the Fokker-Planck collisions is somewhat higher, which correlates with the higher linear gain exponent and 

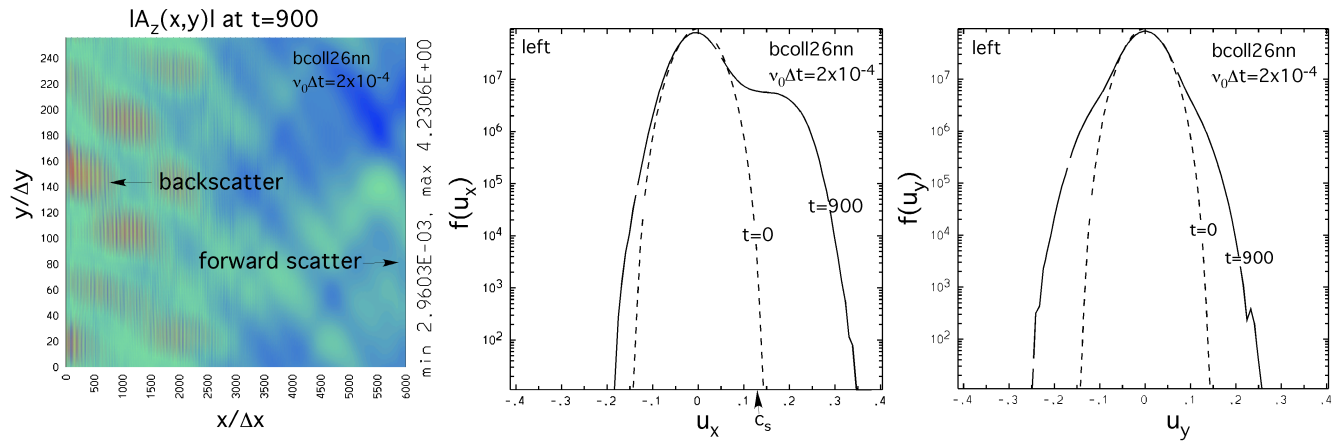

Figure 16. Absolute value of the electromagnetic potential $\left|A_{\mathrm{z}}(x, y)\right|$ at $t=900$, ion velocity distribution functions $f\left(u_{\mathrm{x}}\right)$ and $f\left(u_{\mathrm{y}}\right)$ at $t=0$ and 900 in simulation bcoll26nn for parameters: $Z T_{\mathrm{e}} / T_{\mathrm{i}}=16, \mathrm{v}_{0} / \mathrm{v}_{\mathrm{e}}=0.2, k_{0} \square_{\mathrm{e}}=0.2, m_{\mathrm{Be}} / m_{\mathrm{e}}=$ real mass ratio, $n_{\mathrm{e}} / n_{\mathrm{c}}=0.1, L_{\mathrm{x}} \sim 192 \square_{0}$, $L_{\mathrm{y}} \sim 16 \square_{0}$, and Fokker-Planck collisions with $\square \square t=2 \square 10^{-4}$.
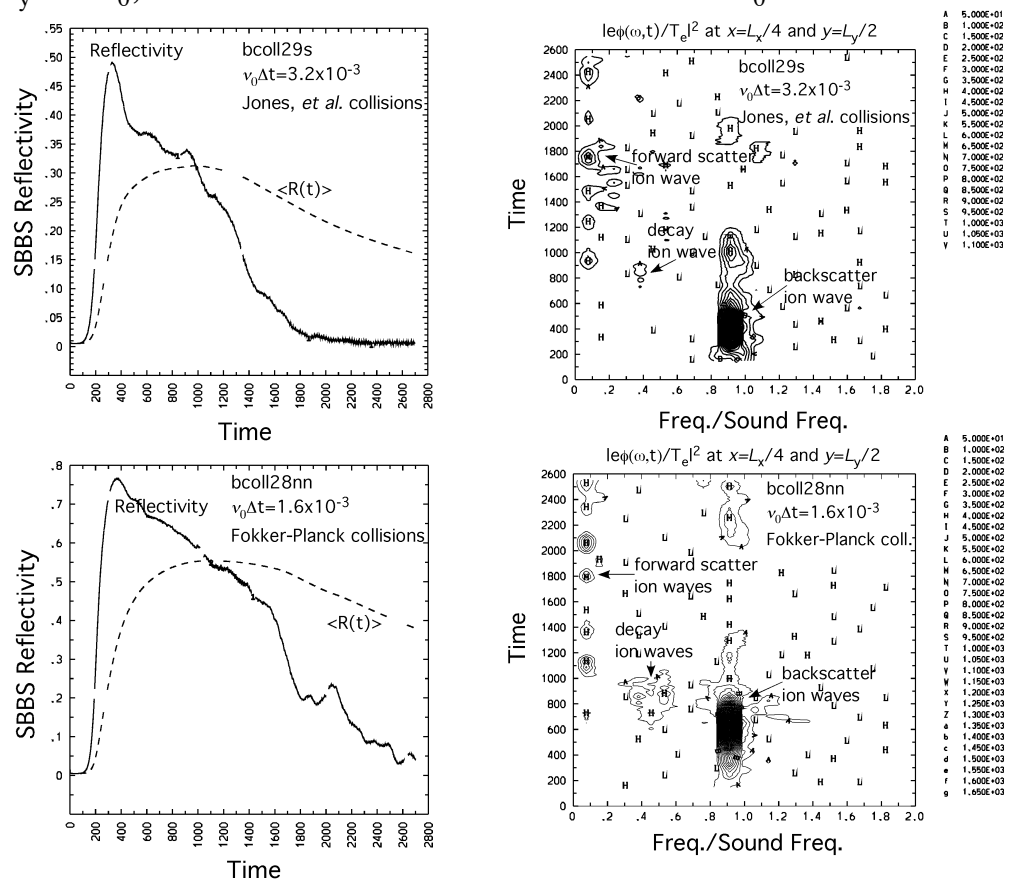

Figure 17. Reflectivities as functions of time and power spectra for $\mid e\left[\left(L_{\mathrm{x}} / 4, L_{\mathrm{y}} / 2\right) /\left.T_{\mathrm{e}}\right|^{2} \mathrm{vs}\right.$. $\square / \square_{\mathrm{s}}$ and time for parameters: $Z T_{\mathrm{e}} / T_{\mathrm{i}}=16, \mathrm{v}_{0} / \mathrm{v}_{\mathrm{e}}=0.2, k_{0} \square_{\mathrm{e}}=0.2, \mathrm{~m}_{\mathrm{Be}} / \mathrm{m}_{\mathrm{e}}=$ real mass ratio, $L_{\mathrm{x}} \sim 192 \square_{0}, L_{\mathrm{y}} \sim 16 \square_{0}, \mathrm{n}_{\mathrm{e}} / \mathrm{n}_{\mathrm{c}}=0.1$, Fokker-Planck model $\left(\square \square \mathrm{t}=1.6 \square 10^{-3}\right.$, bcoll28nn) and Jones, et al. model $\left(\square \square \mathrm{t}=3.2 \square 10^{-3}\right.$, bcoll29s)

the reduced collisionality of the resonant ions, as compared to the simulation with velocity-independent collisions. 
Figures 17 and 18 show results comparing simulations with Fokker-Planck collisions $\left(\square \square \mathrm{t}=1.6 \square 10^{-3}\right.$, bcoll28nn) and Jones, et al. collisions $\left(\square \square \mathrm{t}=3.2 \square 10^{-3}\right.$, bcoll29s $)$ for our baseline parameters. The peak reflectivity with Fokker-Planck collisions is higher than with velocity-independent collisions and correlates with having a higher gain exponent owing to the weaker SBBS ion wave damping rate. In Fig. 17 the reflectivity with Fokker-Planck collisions takes longer to relax, and two-ion-wave decay products are more evident in the streak spectra for $\mathrm{l}\left[\left(L_{\mathrm{x}} / 4, L_{\mathrm{y}} / 2\right) / T_{\mathrm{e}} \mathrm{e}^{2}\right.$. The velocity distribution
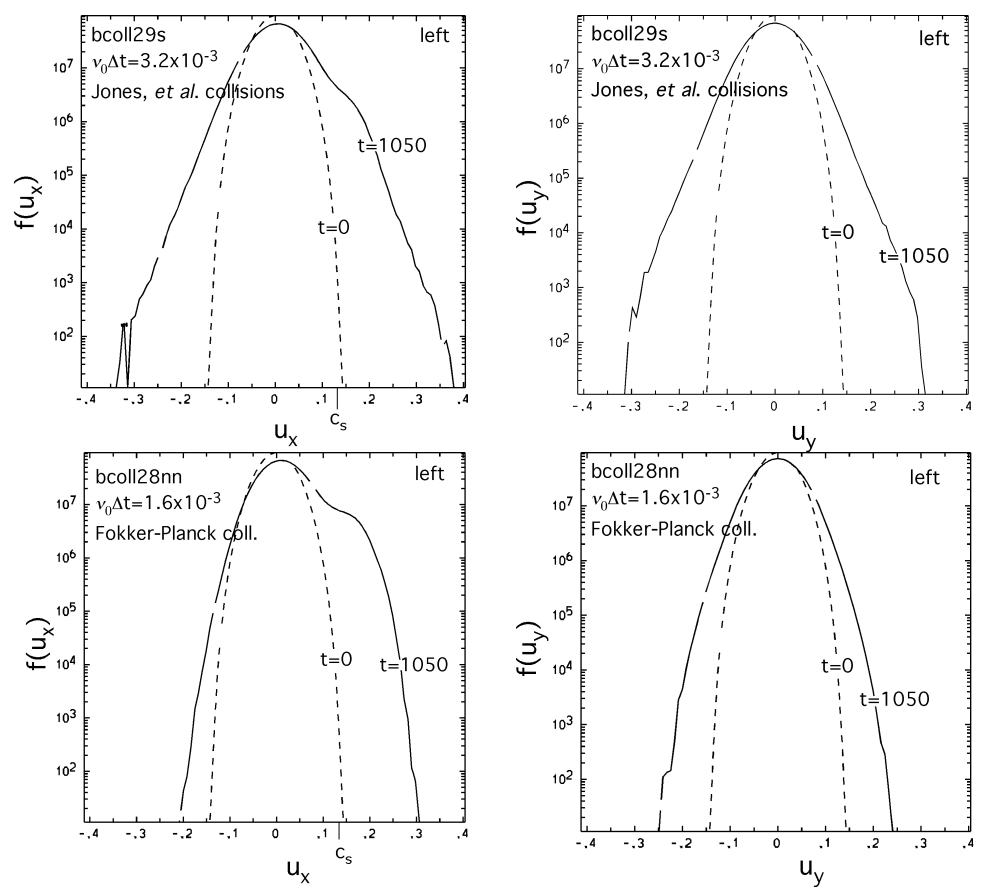

Figure 18. Velocity distribution functions $f\left(u_{\mathrm{x}}\right)$ and $f\left(u_{\mathrm{y}}\right)$ on the left side of the domain at $t=0$ and 1050 for parameters: $Z T_{\mathrm{e}} / T_{\mathrm{i}}=16, \mathrm{v}_{0} / \mathrm{v}_{\mathrm{e}}=0.2, k_{0} \square_{\mathrm{e}}=0.2, m_{\mathrm{Be}} / m_{\mathrm{e}}=$ real mass ratio, $L_{\mathrm{x}} \sim 192 \square_{0}, L_{\mathrm{y}} \sim 16 \square_{0}, n_{\mathrm{e}} / n_{\mathrm{c}}=0.1$, Fokker-Planck model $\left(\square_{0} \square \mathrm{t}=1.6 \square 10^{-3}\right.$, bcoll28nn) and Jones, et al. model $\left(\square \square \mathrm{t}=3.2 \square 10^{-3}\right.$, bcoll29s)

functions at $t=1050$ indicate that the hot ion tail produced by trapping in the SBBS ion wave is thermalized and isotropized to high degree by the Jones, et al. collisions, but much less so by the Fokker-Planck collisions. The effective $Z T_{\mathrm{e}} / T_{\mathrm{i}}$ is lower and there is 
more slope in the $f\left(u_{\mathrm{x}}\right)$ velocity distribution function at the sound speed with the Jones, et al. collisions; hence, the ion Landau damping is higher and the ion trapping nonlinear frequency shift is much smaller than with the Fokker-Planck collisions.

The observations related to Figs. 17 and 18 suggest the following interpretation of the SBBS reflectivity results. Previous analyses have shown that the two-ion-wave decay is sensitive to both the damping rate of the decay products and having the pump ion wave frequency be above its normal mode frequency (possibly nonlinear). ${ }^{1,2}$ Thus, the conditions for the two-ion-wave decay are relatively unfavorable in simulation bcoll29s (Jones, et al. collisions) and more favorable in bcoll28nn (Fokker-Planck collisions). In bcoll29s the strong collisions in combination with the energy deposition from the SBBS ion wave rapidly evolve the velocity distribution function to a state in which there is much less flattening of the $f\left(u_{\mathrm{x}}\right)$ ion velocity distribution, which then reduces the nonlinear frequency shift of the primary SBBS ion wave and much increases its ion Landau damping resulting in quenching the SBBS reflectivity. The two-ion-wave decay becomes subdominant to direct damping of the primary SBBS ion wave as the collisionality is increased in the simulations with the Jones, et al. collisions, which increases the ion Landau damping in both the linear and nonlinear regimes. In contrast, two-ion-wave decay is more evident in the SBBS simulations with Fokker-Planck collisions. While increasing the collisionality increases ion wave damping, decreases the gain exponent, and reduces the peak reflectivity for both collision models in the early part of the simulations, on a longer time scale increased collisionality inhibits the two-ionwave decay because it elevates the damping rate of the decay product ion waves and reduces the nonlinear frequency shift of the primary SBBS ion wave associated with the 
flattening of the ion velocity distribution due to ion trapping. Thus, in the simulations with Fokker-Planck collisions in which the two-ion-wave decay is important, the increased inhibition of the two-ion-wave decay with increased collisionality contributes to increasing the quench time for the SBBS by retarding the relaxation of the primary SBBS ion wave. We conclude that the differences in the importance of the two-ion-wave decay with increasing collisionality in the simulations with the two collision models can account for the differences observed in the SBBS time-averaged reflectivities.

\section{SATURATION OF BRILLOUIN BACKSCATTER WITH A FLOW GRADIENT}

Plasma flow gradients have an important influence on stimulated Brillouin scattering instability and alter both its linear convective gain and the nonlinear saturation. Generally, a linear flow gradient can localize SBBS to a resonance region whose width depends jointly on the dissipation present and the gradient length affecting the frequency and wavenumber matching conditions. Short linear gradient lengths limit the convective gain of the instability. ${ }^{8,32}$ Reference 11 showed that the nonlinear frequency shift due to ion trapping, which increases in the direction of the backscatter convective gain, can partially compensate for the dephasing caused by a spatial gradient in the plasma flow that is parallel to the backscatter direction and thus enhance the SBBS convective amplification nonlinearly ("auto-resonance"). When the flow gradient is oriented opposite to the backscatter direction, then the nonlinear frequency shift due to ion trapping is additive to the dephasing effect of the spatial gradient; and there is a nonlinear “anti-auto-resonant" decrease in the SBBS convective amplification. 
Williams, et al. ${ }^{11}$ considered auto-resonant enhancement and anti-auto-resonant decrease in the stimulated Brillouin scattering driven cross-beam interaction (where two incident lasers optically mix in the plasma flow and produce a beat-wave that resonantly excites an ion wave at the Doppler-shifted beat frequency and beat wavenumber of the two lasers). Analytical and numerical results were obtained for the steady-state crossbeam amplification with the ion wave damping rate a specified parameter. ${ }^{11}$ The analysis shows that finite auto-resonant or anti-auto-resonant effects can be expected when the magnitude of the ion trapping nonlinear frequency shift is competitive with the ion wave damping rate, and these effects are stronger the larger the linear convective amplification is (see Fig. 4 of Ref. 11).

We performed a series of collisionless 2D SBBS simulations with flow gradients either parallel or anti-parallel to the backscatter direction. The backscatter grew from noise to large amplitude, saturated with ion trapping and ion wave decay effects evident, and relaxed to minuscule reflectivities. The ion wave damping rates (estimated by taking the ratio of the time derivative of the ion kinetic energy divided by twice the ion wave energy density integrated over the volume) varied in time as the ion velocity distribution function evolved. Thus, comparing the steady-state analytical theory of Williams, et al. ${ }^{11}$ directly to these SBBS simulations is not feasible for several reasons. However, we can use the Williams, et al., calculations to deduce in what circumstance auto-resonant and anti-auto-resonant effects are expected and then look for these effects in the simulations. A measure of the strength of the ion trapping frequency shift is given by $\hat{\square} \sim\left(\square \square_{\text {trap }} / \square_{s}\right)^{4}$ where $\square$ is the SBBS ion wave damping rate and $\square \square_{\text {trap }}=\square 0.1\left(\left[n_{e} / n_{e}\right)^{1 / 2} \square_{s} \square \square 0.003 \square_{s}\right.$ is the ion trapping frequency shift for $Z T_{\mathrm{e}} / T_{\mathrm{i}}=16$ 
and the peak ion wave amplitudes observed. For $1 \square 10^{-3} \leq \square \square_{s} \leq 2 \square 10^{-3}$ observed near the temporal peak of the SBBS reflectivity, $\hat{\square} \sim 10^{3} \square 10^{4}$ in our simulations. For the baseline parameters defined earlier and with $\left(L_{x} / 2\right) d\left(V_{d r i f t} / c_{s}\right) / d x= \pm\{0.05,0.1,0.2\}$ for the flow gradients, the linear convective gain exponent for intensity is $G_{\text {SBBS }}^{\mathrm{I}}=5-20$, where

$$
G_{S B B S}^{I}=\frac{1}{8} \frac{\mathrm{v}_{0}^{2}}{\mathrm{v}_{e}^{2}} \frac{n_{e}}{n_{c}} \frac{\square_{s}}{\square_{s}} \frac{\square_{0} \square\left|L_{\mathrm{v}}\right|}{v_{g}\left(1+k^{2} \square_{e}^{2}\right)}
$$

and $L_{\mathrm{V}}=(d \operatorname{Re} \square / d x)^{\square 1}=c_{s}\left(2 d V_{d r i f t} / d x\right)^{\square 1} .8,11,32$ Thus, we expect auto-resonant and antiauto-resonant effects to be evident in the simulations for the given parameters.

Figure 19 shows the results of the series of 2D collisionless SBBS simulations with flow gradients and the baseline parameters. One series plotted used $v_{0} / v_{e}=0.2$ with five-point-stencil digital smoothing of the ion charge density, and the other series used $\mathrm{v}_{0} / \mathrm{v}_{\mathrm{e}}=0.15$ with successive three-point stencil digital smoothing in $x$ and $y$ which gave a lower amplitude noise source for the backscattering. The former series with higher incident pump power has higher gain exponents and reflectivities for the same flow gradient. With flow gradients parallel to the backscatter direction (negative $x$ ), there is auto-resonant enhancement of the SBBS reflectivities relative to those for the anti-autoresonant orientation of the flow gradient, except at the lowest values of intensity gain exponent where the theory in Ref. 11 indicates that there should be little or no autoresonant enhancement of the reflectivities. The peak reflectivities are affected by pump depletion, which limits the enhancement due to auto-resonance. 

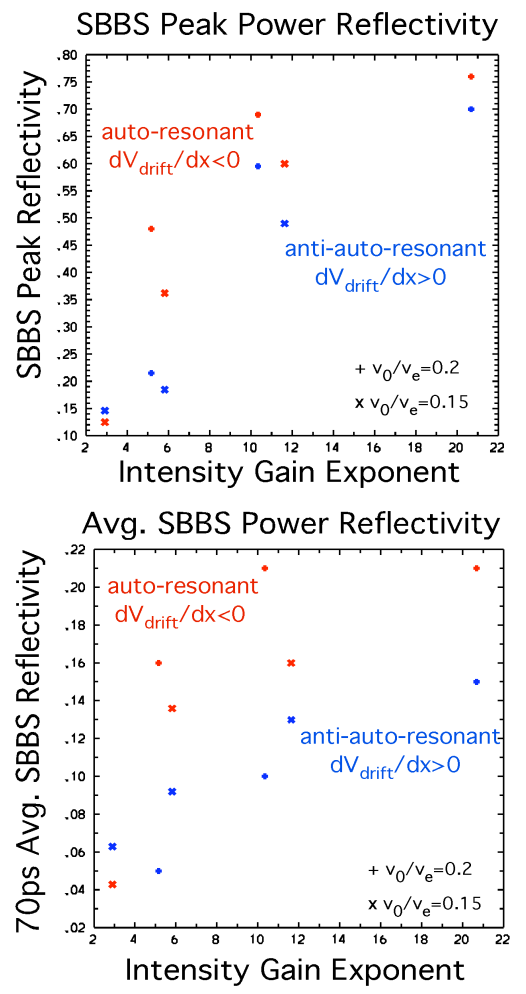

Figure 19. Peak and 70ps average reflectivities vs. linear convective gain exponents for backscatter intensity with parameters: $Z T_{\mathrm{e}} / \mathrm{T}_{\mathrm{i}}=16, k_{0} \square_{\mathrm{e}}=0.2, \mathrm{v}_{0} / \mathrm{v}_{\mathrm{e}}=0.15$ and 0.2 , $m_{\mathrm{Be}} / m_{\mathrm{e}}=$ real mass ratio, $n_{\mathrm{e}} / n_{\mathrm{c}}=0.1$, collisionless, $\left(L_{\mathrm{x}} / 2 c_{\mathrm{s}}\right) d V_{\mathrm{drift}} / d x= \pm(0.05,0.1,0.2)$

In Figures 20 and 21 we show results from a representative simulation (bgradv5d) with a linear flow gradient directed parallel to the backscatter direction. As in the collisionless and collisional simulations with no flow, ion trapping produces a hot ion tail; and ion-wave decay and trapping saturate SBBS, while forward SBS occurs later in time with the largest amplitudes on the right side of the simulation box. Ion heating in $u_{\mathrm{y}}$ results from the obliquely propagating decay ion waves accompanying the quench of the SBBS. 

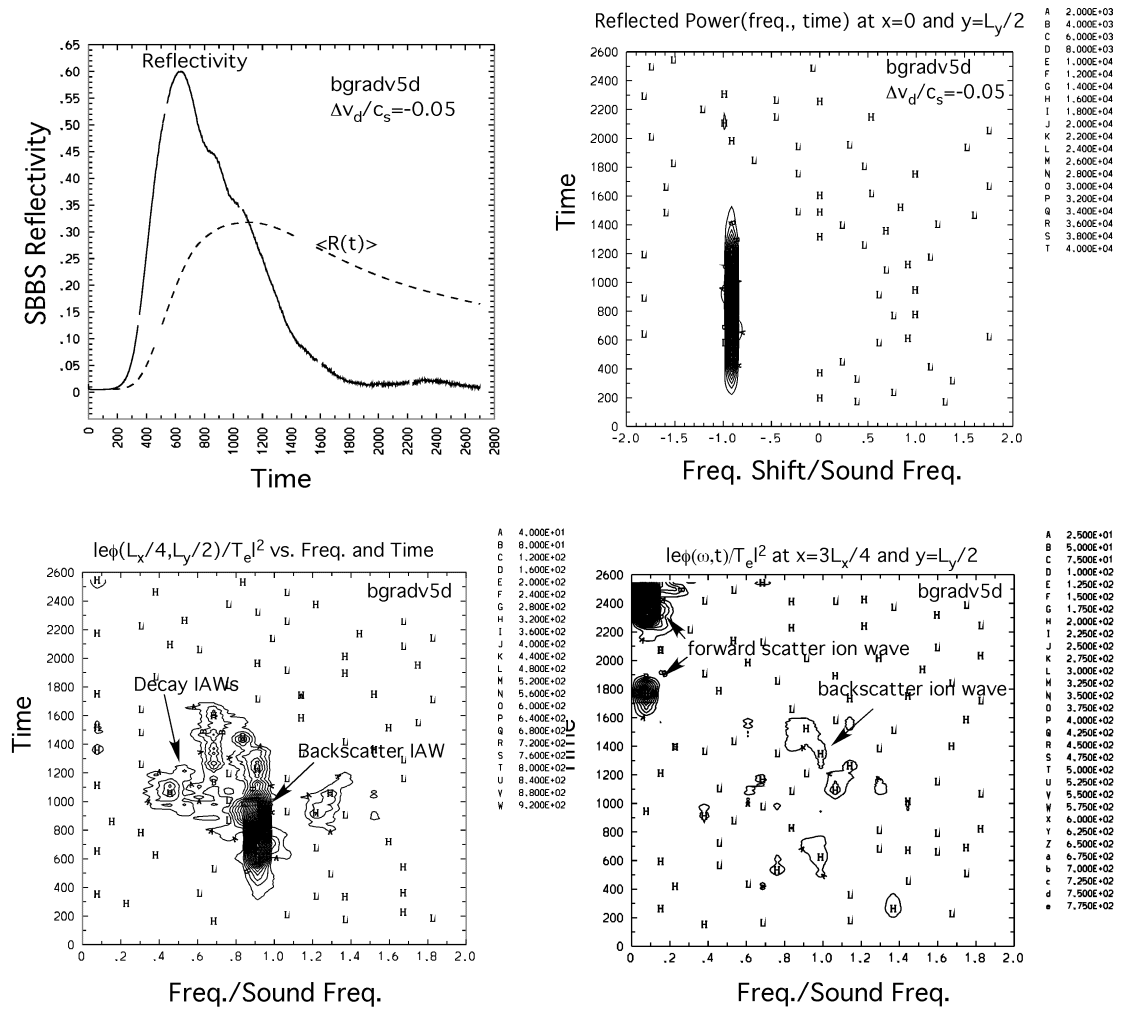

Figure 20. SBBS instantaneous reflectivity and cumulative time-average reflectivity vs. time, and power spectra plotted as functions of frequency and time for the reflected electromagnetic power at $x=0$ and $y=L_{\mathrm{y}} / 2$, $\left.\mathrm{e} e \square \square_{\mathrm{e}}\right|^{2}$ at $x=L_{\mathrm{x}} / 4$ and $y=L_{\mathrm{y}} / 2$, and $\left|e \square \square \square_{\mathrm{e}}\right|^{2}$ at $x=3 L_{\mathrm{x}} / 4$ and $y=L_{\mathrm{y}} / 2$, for parameters: $Z T_{\mathrm{e}} / T_{\mathrm{i}}=16, \mathrm{v}_{0} / \mathrm{v}_{\mathrm{e}}=0.2, k_{0} \square_{\mathrm{e}}=0.2, m_{\mathrm{Be}} / m_{\mathrm{e}}=$ real mass ratio, $n_{\mathrm{e}} / n_{\mathrm{c}}=0.1$, no ion collisions, and linear velocity gradient $\left(L_{\mathrm{x}} / 2 c_{\mathrm{s}}\right) d \mathrm{~V}_{\text {drift }} / d x=-0.05$
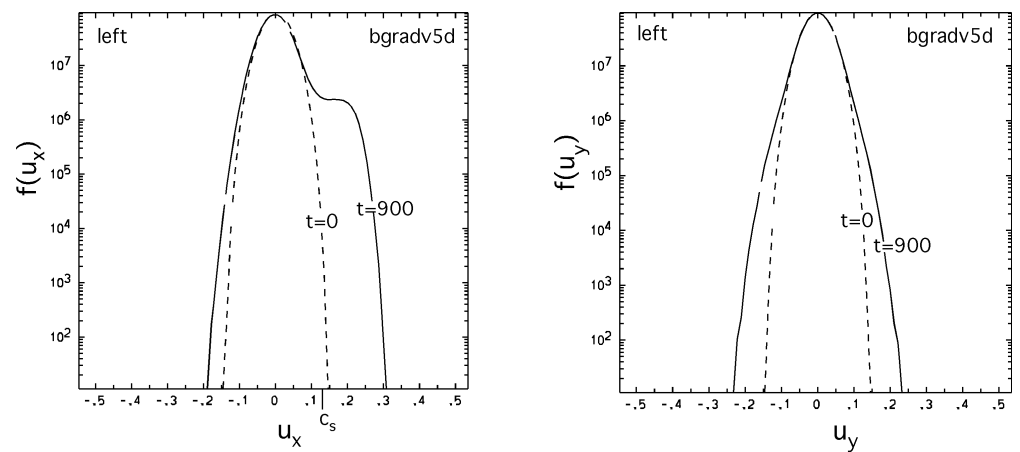

Figure 21. Ion velocity distribution functions $f\left(u_{\mathrm{x}}\right)$ and $f\left(u_{\mathrm{y}}\right)$ at $t=0$ and 900 for simulation bgradv5d with linear velocity gradient $\left(L_{\mathrm{x}} / 2 c_{\mathrm{s}}\right) d / \mathrm{v}_{\text {drift }} / d x=-0.05$ showing formation of a hot ion tail and transverse heating. 


\section{CONCLUSIONS}

We have reported two-dimensional simulations with kinetic ions addressing the effects of ion-ion collisions and plasma flow gradients on the saturation of stimulated Brillouin backscattering. Ion-ion collisions have been implemented in the BZOHAR simulation code using two different Langevin-equation algorithms, one with velocityindependent collisions ${ }^{23}$ and the other with a Fokker-Planck collision operator in three velocity dimensions. ${ }^{24,25}$ Both algorithms use the ion density, mean velocity, and temperature accumulated from the evolving ion particle distribution function locally in space, but are simplified by assuming that the multi-dimensional ion velocity distribution is close to an isotropic Maxwellian. The latter assumption is violated by the formation of a hot-ion tail in $f\left(u_{x}\right)$ due to trapping of ions in the SBBS ion waves; however, the transverse ion velocity distribution functions remain close to an isotropi Maxwellian. Over time the hot ion tail is thermalized by the collisions; and the ion velocity distribution function can relax toward an isotropic Maxwellian at a higher temperature after the SBBS quenches.

Brillouin backscatter peak reflectivities are reduced in the presence of ion-ion collisions. Ion collisions are another source of dissipation for the ion waves, restore slope to the velocity distribution function (flattened due to trapping), and, as stated, thermalize the hot ion tail formed by trapping in the SBS ion wave (effectively increasing $Z T_{\mathrm{e}} / T_{\mathrm{i}}$ ), as pointed out in earlier work. ${ }^{18}$ Increased damping of the SBBS ion wave assists in reducing the peak backscatter reflectivities. Ion trapping and secondary ion wave instabilities remain important saturation mechanisms for strong $\mathrm{SBBS}, Z T_{\mathrm{e}} / T_{\mathrm{i}}>>1$, and the range of collisionality investigated with the more physical Fokker-Planck collision model. The two-ion-wave decay instability is relatively more important with increasing 
ion collisionality in the simulations with the Fokker-Planck collision model than in the simulations with the Jones, et al., velocity-independent, collision model, which influences the relaxation of SBBS and the time-averaged reflectivities.

SBS backscatter simulations including a spatial gradient in the plasma flow velocity have been performed that show increases in the reflectivity due to auto-resonant effects (relative to anti-auto-resonant effects) associated with the interplay of the nonlinear frequency shift due to ion trapping with the linear dephasing effect of a spatial gradient in the plasma flow velocity. The simulations with flow gradients give further evidence of the robustness of the SBBS saturation mechanism involving ion trapping and secondary ion wave instabilities in two dimensions for strong SBBS, $Z T_{\mathrm{e}} / T_{\mathrm{i}} \gg>1$, and conditions of interest for current laser-fusion experiments. ${ }^{32}$

We gratefully acknowledge the many contributions to this effort and encouragement from Barbara Lasinski, Wally Manheimer, Richard Berger, Dustin Froula, Siegfried Glenzer, Denise Hinkel, Robert Kirkwood, Bill Kruer, Peter Rambo, Bert Still, and Larry Suter. This work was performed under the auspices of the U.S. Dept. of Energy by the University of California Lawrence Livermore National Laboratory under contract No. W-7405-ENG-48. 


\section{References}

${ }^{1}$ B.I. Cohen, B.F. Lasinski, A.B. Langdon, and E.A. Williams, Phys. Plasmas 4, 956 (1997).

${ }^{2}$ B.I. Cohen, L. Divol, A. B. Langdon, and E. A. Williams, Phys. Plasmas 12, 052703 (2005).

${ }^{3}$ B.I. Cohen, B.F. Lasinski, A.B.Langdon, E.A. Williams, K.B. Wharton, R.K. Kirkwood, and K. G. Estabrook, Phys. Plasmas 5, 3408 (1998).

${ }^{4}$ C. Riconda, S. Huller, J. Myatt, and D. Pesme, Phys. Scr. T 84, 217 (2000).

${ }^{5}$ E.A. Williams, Bull. Amer. Phys. Soc. 42, 1998 (1997).

${ }^{6}$ D.W. Forslund, J.M. Kindel, and E.L. Lindman, Phys. Fluids 18, 1017 (1975).

${ }^{7}$ W.L. Kruer, Phys. Fluids 23, 1273 (1980).

${ }^{8}$ W.L. Kruer, The Physics of Laser Plasma Interactions (Addison-Wesley, Reading, MA, 1988).

${ }^{9}$ A. A. Andreev and V.T. Tikhonchuk, Sov. Phys. JETP 68, 1135 (1989).

${ }^{10}$ H.X. Vu, J. Comput. Phys. 124, 417 (1996); H.X. Vu, Phys. Plasmas 4, 1841 (1997);

R.E. Giacone and H.X. Vu, Phys. Plasmas 5, 1455 (1998).

${ }^{11}$ E.A. Williams, B.I. Cohen, L. Divol, M.R. Dorr, J.A. Hittinger, D.E. Hinkel, A.B.

Langdon, R. K. Kirkwood, D.H. Froula, and S.H. Glenzer, Phys. Plasmas 11, 231 (2004).

${ }^{12}$ L. Divol, B.I. Cohen, E.A. Williams, A.B. Langdon, B.F. Lasinski, Phys. Plasmas 10, 3728 (2003); L. Divol, R.L. Berger, B.I. Cohen, E.A. Williams, A.B. Langdon, B.F. Lasinski, D.H. Froula, and S.H. Glenzer, Phys. Plasmas 10, 1822 (2003).

${ }^{13}$ J.A. Heikkinen, S.J. Kartunen, R.R.E. Salomaa, Phys. Fluids 27, 707 (1984).

${ }^{14}$ K. Estabrook, W.L. Kruer, and M.G. Haines, Phys. Fluids B 1, 1282 (1989). 
${ }^{15}$ A.V. Maximov, W. Rozmus, V.T. Tikhonchuk, D.F. Dubois, H.A. Rose, and A.M.

Rubenchik, Phys. Plasmas 3, 1689 (1996).

${ }^{16}$ C. Riconda, A. Heron, D. Pesme, S. Huller, V. Tikhonchuk, and F. Detering, Phys. Rev. Lett. 94, 055003 (2005).

${ }^{17}$ S. Weber, C. Riconda, and V.T. Tikhonchuk, Phys. Rev. Lett. 94, 055005 (2005).

${ }^{18}$ P.W. Rambo, S.C. Wilks, and W.L. Kruer, Phys. Rev. Lett. 79, 83 (1997).

${ }^{19}$ S.H. Glenzer, L. Divol. R.L. Berger, C. Geddes, R.K. Kirkwood, J.D. Moody, E.A.

Willams, and P.E. Young, Phys. Rev. Lett 86, 2565 (2001).

${ }^{20}$ D.H. Froula, L. Divol, and S.H. Glenzer, Phys. Rev. Lett. 88, 105003 (2002); D.H.

Froula, L. Divol, H.A. Baldis, R.L. Berger, D.G. Braun, B.I. Cohen, R.P. Johnson, D.S.

Montgomery, E.A. Williams, et al., Phys. Plasmas 9, 4709 (2002); D.H. Froula, L.Divol,

D.G. Braun, B.I. Cohen, G. Gregori, A. Mackinnon, E.A. Willams, S.H. Glenzer, H.A.

Baldis, D.S. Montgomery, et al., Phys. Plasmas 10, 3728 (2003).

${ }^{21}$ H.C. Bandulet, C. Labaune, K. Lewis, and S. Depierreux, Phys. Rev. Lett. 93, 035002 (2004).

${ }^{22}$ C. Niemann, S.H. Glenzer, J. Knight, L. Divol, B.I. Cohen, C. Constantin, D.H. Froula, E.A. Williams, D.S. Montgomery, Phys. Rev. Lett. 93, 0045004 (2004).

${ }^{23}$ M.E. Jones, D. S. Lemons, R. J. Mason, V. A. Thomas, and D. Winske, J. Comp. Phys. 123, 169 (1996).

${ }^{24}$ T.D. Rognlien and T.A. Cutler, Nuc. Fusion 20, 1003 (1980).

${ }^{25}$ W.M. Manheimer, M. Lampe, and G. Joyce, J. Comp. Phys. 138, 565 (1997).

${ }^{26}$ D. S. Lemons, J. Lackman, M.E. Jones, and D. Winske, Phys. Rev. E 52, 6855 (1995).

${ }^{27}$ C.K. Birdsall and A.B. Langdon, Plasma Physics Via Computer Simulation (McGraw- 
Hill, New York, 1985).

${ }^{28}$ P.W. Rambo, J. Comp. Phys. 133, 173 (1997).

${ }^{29}$ B.A. Trubnikov, Reviews of Plasma Physics (Consultant's Bureau, New York, 1965), Vol. 1, p. 105.

${ }^{30}$ C.J. Randall, Phys. Fluids 25, 2231 (1982).

${ }^{31}$ G..J. Morales and T.M. O’Neil, Phys. Rev. Lett. 28, 417 (1972); H. Ikezi, K.

Scharzeneggar, A.L. Simons, Y. Ohsawa, T. Kamimura, Phys. Fluids 21, 239 (1978).

${ }^{32}$ J.D. Lindl, P. Amendt, R.L. Berger, S. G. Glendinning, S.H. Glenzer, S.W. Haan, R.L.

Kauffman, O.L. Landen, L.J. Suter, Physics of Plasmas 11, 339 (2004). 